\title{
Quasi-linear parabolic equations with degenerate coercivity having a quadratic gradient term
}

\section{Équations paraboliques quasi-linéaires avec coercivité dégénérée et un terme à croissance quadratique par rapport au gradient}

\author{
A. Dall'Aglio ${ }^{\text {a,* }}$, D. Giachetti ${ }^{\text {a }}$, C. Leone ${ }^{b}$, S. Segura de León ${ }^{\text {c }}$ \\ a Dipartimento di Metodi e Modelli Matematici - Università di Roma "La Sapienza”, Via Antonio Scarpa 16, 00161 Roma, Italy \\ b Dipartimento di Matematica e Applicazioni "R. Caccioppoli”, Università di Napoli "Federico II", Via Cintia, Monte S. Angelo, \\ 80126 Napoli, Italy \\ ${ }^{\mathrm{c}}$ Departament d'Anàlisi Matemàtica - Universitat de València, Dr. Moliner 50, 46100 Burjassot, València, Spain
}

Received 17 February 2004; received in revised form 24 November 2004; accepted 3 February 2005

Available online 15 July 2005

\begin{abstract}

$$
\begin{cases}u_{t}-\operatorname{div}(\alpha(u) \nabla u)=\beta(u)|\nabla u|^{2}+f(x, t), & \text { in } \Omega \times] 0, T[ \\ u(x, t)=0, & \text { on } \partial \Omega \times] 0, T[ \\ u(x, 0)=u_{0}(x), & \text { in } \Omega .\end{cases}
$$
\end{abstract}

We study existence and regularity of distributional solutions for possibly degenerate quasi-linear parabolic problems having a first order term which grows quadratically in the gradient. The model problem we refer to is the following

Here $\Omega$ is a bounded open set in $\mathbb{R}^{N}, T>0$. The unknown function $u=u(x, t)$ depends on $x \in \Omega$ and $\left.t \in\right] 0, T$. The symbol $\nabla u$ denotes the gradient of $u$ with respect to $x$. The real functions $\alpha, \beta$ are continuous; moreover $\alpha$ is positive, bounded and may vanish at $\pm \infty$. As far as the data are concerned, we require the following assumptions:

$$
\int_{\Omega} \Phi\left(u_{0}(x)\right) \mathrm{d} x<\infty
$$

\footnotetext{
* Corresponding author.

E-mail addresses: aglio@dmmm.uniroma1.it (A. Dall'Aglio), giachetti@dmmm.uniroma1.it (D. Giachetti), chileone@unina.it (C. Leone), sergio.segura@uv.es (S. Segura de León).
}

0294-1449/\$ - see front matter (C) 2006 L'Association Publications de l'Institut Henri Poincaré. Published by Elsevier B.V. All rights reserved doi:10.1016/j.anihpc.2005.02.006 
where $\Phi$ is a convenient function which is superlinear at $\pm \infty$ and

$$
f(x, t) \in L^{r}\left(0, T ; L^{q}(\Omega)\right) \quad \text { with } \frac{1}{r}+\frac{N}{2 q} \leqslant 1 .
$$

We give sufficient conditions on $\alpha$ and $\beta$ in order to have distributional solutions. We point out that the assumptions on the data do not guarantee in general the boundedness of the solutions; this means that the coercivity of the principal part of the operator can really degenerate. Moreover, a boundedness result is proved when the assumptions on the data are strengthened.

(C) 2006 L'Association Publications de l'Institut Henri Poincaré. Published by Elsevier B.V. All rights reserved

\section{Résumé}

Nous étudions l'existence et la régularité des solutions au sens des distributions de problèmes paraboliques quasi-linéaires qui présentent un terme du premier ordre à croissance quadratique par rapport au gradient et dont la partie principale peut dégénérer.

Le problème modèle auquel nous nous référons est (1) ci-dessous, où les fonctions $\alpha$ et $\beta$ sont à valeurs réelles et continues ; de plus $\alpha$ est positive et bornée mais peut s'annuler à $\pm \infty$. En ce qui concerne les données $u_{0}(x)$ et $f(x, t)$, nous supposons que $\int_{\Omega} \Phi\left(u_{0}(x)\right) \mathrm{d} x<\infty$, où la fonction $\Phi$ est superlinéaire à $\pm \infty$, et que $f(x, t) \in L^{r}\left(0, T ; L^{q}(\Omega)\right)$ avec $\frac{1}{r}+\frac{N}{2 q} \leqslant 1$.

Nous donnons des conditions suffisantes sur $\alpha$ et $\beta$ qui assurent l'existence de solutions au sens des distributions. Ces conditions sur les données n'impliquent pas en général que les solutions soient bornées, donc la coercivité de la partie principale de l'opérateur peut vraiment dégénérer. Mais quand nous imposons des conditions plus fortes sur les données, nous démontrons que les solutions sont bornées.

(C) 2006 L'Association Publications de l'Institut Henri Poincaré. Published by Elsevier B.V. All rights reserved

MSC: 35K55; 35K65; 35B45; 35K20

Keywords: Nonlinear parabolic problems; Gradient term with quadratic growth; Existence and regularity; Bounded and unbounded solutions;

Lack of coerciveness

\section{Introduction}

Our aim is to study existence for a class of quasi-linear parabolic problems involving first order terms with natural growth with respect to the gradient. The model problem we refer to is (1) above, where $\Omega$ is a bounded open set in $\mathbb{R}^{N}, T>0$, and $u=u(x, t)$, with $x \in \Omega$ and $\left.t \in\right] 0, T[$.

Let us remark that, if the functions $\alpha, \beta$ are bounded on the real line, and $\alpha(s) \geqslant \alpha_{0}>0$ for every $s \in \mathbb{R}$ (i.e., if the principal part is assumed to be uniformly coercive), in the case where the initial datum $u_{0}(x)$ belongs to ${ }^{1}$ $L^{\infty}(\Omega)$ and

$$
f(x, t) \in L^{r}\left(0, T ; L^{q}(\Omega)\right), \quad \frac{q(r-1)}{r}>\frac{N}{2},
$$

it is possible to prove existence of bounded weak solutions for problem (1) (see, for instance, [8,24,25] and [13]). Recently, in the case where $\alpha$ may vanish at infinity, Boccardo and Porzio (see [9]) assume that $\alpha(s)$ and $\beta(s)$ satisfy

$$
\alpha \in L^{\infty}(\mathbb{R}), \quad \alpha \notin L^{1}(-\infty, 0) \cup L^{1}(0,+\infty), \quad \frac{\beta}{\alpha} \in L^{1}(\mathbb{R}) .
$$

Then, if $u_{0} \in L^{\infty}(\Omega)$ and $f(x, t) \in L^{m}(\Omega \times] 0, T\left[\right.$ ), with $m>1+\frac{N}{2}$ (which is a very special case of condition (2)), they prove the existence of bounded weak solutions for problem (1).

\footnotetext{
1 The symbols $L^{q}(\Omega), L^{r}\left(0, T ; L^{q}(\Omega)\right)$, and so forth, denote the usual Lebesgue spaces, see for instance [11] or [16]. Moreover we will sometimes use the shorter notations $\|f\|_{q},\|f\|_{r, q}$ instead of $\|f\|_{L^{q}(\Omega)},\|f\|_{L^{r}\left(0, T ; L^{q}(\Omega)\right)}$, respectively. The symbol $H_{0}^{1}(\Omega)$ denotes the Sobolev space of functions with distributional derivatives in $L^{2}(\Omega)$ which have zero trace on $\partial \Omega$. $H^{-1}(\Omega)$ denotes the dual space of $H_{0}^{1}(\Omega)$. The spaces $L^{2}\left(0, T ; H_{0}^{1}(\Omega)\right)$ and $L^{2}\left(0, T ; H^{-1}(\Omega)\right)$ have obvious meanings, see again [11] or [16].
} 
In the present paper we are interested in finding more general conditions on $\alpha$ and $\beta$ (which include (3)) and also in dealing with the case where the integrability of the data $u_{0}$ and $f$ is not so high to allow bounded solutions. The assumptions for the model problem are the following: if we define

$$
\gamma(s)=\int_{0}^{s} \frac{\beta(\sigma)}{\alpha(\sigma)} \mathrm{d} \sigma, \quad \Psi(s)=\int_{0}^{s} \alpha(\sigma) \mathrm{e}^{|\gamma(\sigma)|} \mathrm{d} \sigma,
$$

we will require, instead of $\beta / \alpha \in L^{1}$, the weaker hypothesis

$$
\mathrm{e}^{|\gamma(s)|} \leqslant C(1+|\Psi(s)|)
$$

for all $s \in \mathbb{R}$. To better understand the role of this assumption on the function $\Psi$, let us first consider the model case where $\alpha=1$ and $f \geqslant 0$; as pointed out, for instance, in [17] (for the stationary problem with constant $\alpha$ and $\beta$ ), we can perform the change of unknown function $v=\Psi(u)$, obtaining the following equation for $v$

$$
v_{t}-\Delta v=f \mathrm{e}^{\gamma(u)}
$$

that under the assumption (4) gives

$$
v_{t}-\Delta v \leqslant C f(1+v),
$$

for which it is not too difficult to obtain some a priori estimates, under suitable assumptions on the data. In the general case, that is, if the operator has the same growth as in the model case, but has a more complicated structure, it is not possible to perform such a change of variable, therefore we need to use suitable exponential test functions related to $\Psi$ and $\gamma$ which allow to get rid of the gradient term and to obtain estimates on the function $u$. We remark that, in the case $\alpha \equiv 1$ (for simplicity), condition (4) is satisfied if $\beta$ is bounded or if $\beta$ is integrable, but it is a more general assumption (see Remark 2.1 below). We point out that for this class of problems the regularity of the data $u_{0}$ and $f$ plays an important role. Indeed, if they have enough integrability (same as in (2)), we will prove the existence of bounded solutions. In this case, the coercivity of the operator is a posteriori not really degenerate. The case where the equality of the exponents in (2) holds is more difficult, because one cannot expect bounded solutions, therefore an actual degeneration of the operator takes place. In this case, we prove the existence of solutions $u$ such that $\Psi(u)$ belongs to the so called energy spaces, that is,

$$
\Psi(u) \in L^{\infty}\left(0, T ; L^{2}(\Omega)\right) \cap L^{2}\left(0, T ; H_{0}^{1}(\Omega)\right) .
$$

Actually, if the initial datum $u_{0}$ is regular enough, one can prove that all powers of $\Psi(u)$ are in these same spaces. If $f$ is less regular than that, i.e., if the opposite inequality holds in (2), the problem of existence is open, even in the uniformly coercive case, since it is not possible to use exponential functions to get rid of the quadratic term (or equivalently, because after change of unknown function, one obtains the inequality (5), for which no a priori estimates hold under these assumptions on $f$ ). The existence result is achieved by approximating the principal part of the equation with uniformly coercive operators, and by truncating the first order term. The first aim is to find a priori estimates on the solutions $u_{n}$ of the approximate problems. Then one has to show that, up to subsequences, $u_{n}$ converges strongly to some function $u$. To this aim, one would like to employ a compactness result of Aubin type (see [4] and [29]), but our estimates do not allow to do this directly, since the function $\Psi$ may have a very weak growth (see, for instance, Remark 2.6). Therefore, we prove a compactness result (see Proposition 6.1 and Corollary 6.1) which apply in this case, and whose proof has been suggested by a similar result by Alt and Luckhaus (see [1]). Then it is necessary to prove pointwise convergence of the gradients of $u_{n}$. This is the most technical part of the paper, and uses an approximating technique to deal with the time derivative of $u_{n}$, previously used in $[22,23,14,13,6,26]$. We point out that no sign assumption is made on the nonlinear first order term throughout the paper. If a "good" sign condition is assumed in the first order nonlinearity (more precisely that this term has the opposite sign of $u$ ), existence of unbounded solutions in the uniformly coercive case is proved in [23] and [14], under weaker assumptions on the data. In a forthcoming paper the corresponding problem for nonlinear operators 
of $p$-Laplace type will be investigated. Moreover weaker assumptions on the operator will be considered, which will lead quite naturally to the use of the notion of entropy solution introduced in [5,28,2]. As far as the stationary problem associated to (1) is concerned, the uniformly coercive case has been studied in many papers (see for instance $[7,17,12,18]$ and references therein). In the case where $\alpha(s)$ may vanish at infinity, which corresponds to a degenerate coercivity of the principal part, existence and regularity results are proved in [10] and [27].

The plan of the paper is the following. Section 2 is devoted to the statement of the assumptions and of the main results. In Section 3 we recall some useful results and we define the approximating problems. In Section 4 we give a priori estimates for the corresponding approximate solutions under the assumptions of the main existence result, Theorem 2.1. Section 5 is devoted to an a priori $L^{\infty}$ estimate under the stronger hypotheses (22) and (23). Finally, Section 6 deals with the limiting process.

\section{Assumptions and main results}

Before stating more precisely our problem, we introduce some notation. We recall that $\Omega$ is a bounded open set in $\mathbb{R}^{N}$, and that $T$ is a positive number. We will denote $\left.\Omega \times\right] 0, T$ [ by $Q_{T}$ and $\left.\partial \Omega \times\right] 0, T\left[\right.$ by $\Sigma_{T}$. We define, for $k>0$, the usual truncation function at level $\pm k$, i.e.,

$$
T_{k}(s)=\max \{-k, \min \{k, s\}\}
$$

and $G_{k}(s)=s-T_{k}(s)=(s-k)^{+} \operatorname{sign}(s)$. Throughout this paper, $C$ will always denote a positive constant which only depends on the parameters of our problem; its value may be different from line to line.

We are interested in studying the following quasilinear evolution problem

$$
\begin{cases}u_{t}-\operatorname{div}(\mathbf{a}(x, t, u) \nabla u)=b(x, t, u, \nabla u)+f, & \text { in } Q_{T} \\ u(x, t)=0, & \text { on } \Sigma_{T} \\ u(x, 0)=u_{0}, & \text { in } \Omega\end{cases}
$$

where the operators satisfy the following hypotheses:

Assumptions on a. The function a: $Q_{T} \times \mathbb{R} \rightarrow \mathbb{R}^{N^{2}}$ satisfies the Carathéodory conditions; that is, it is measurable with respect to $(x, t)$ for all $s \in \mathbb{R}$ and continuous in $s$ for almost all $(x, t) \in Q_{T}$; moreover it satisfies the following assumptions

(A1) There exists a bounded continuous positive function $\alpha: \mathbb{R} \rightarrow \mathbb{R}$ such that

$$
\alpha \notin L^{1}(0,+\infty) \cup L^{1}(-\infty, 0),
$$

and that

$$
(\mathbf{a}(x, t, s) \xi, \xi) \geqslant \alpha(s)|\xi|^{2}
$$

for almost all $(x, t) \in Q_{T}$ and all $(s, \xi) \in \mathbb{R} \times \mathbb{R}^{N}$.

(A2) There exists $C_{0}>1$ such that

$$
|\mathbf{a}(x, t, s) \xi| \leqslant C_{0} \alpha(s)|\xi|
$$

for almost all $(x, t) \in Q_{T}$ and all $(s, \xi) \in \mathbb{R} \times \mathbb{R}^{N}$.

For brevity of notation, we will sometimes write $\mathbf{a}(x, t, s) \xi \xi$ instead of $(\mathbf{a}(x, t, s) \xi$, $\xi)$. Moreover we will often omit the explicit dependence of $\mathbf{a}$ on $x$ and $t$, writing $\mathbf{a}(s)$ instead of $\mathbf{a}(x, t, s)$.

Assumptions on $b$. The function $b: Q_{T} \times \mathbb{R} \times \mathbb{R}^{N} \rightarrow \mathbb{R}$ satisfies the Carathéodory conditions and moreover: 
(B1) There exists a continuous nonnegative function $\beta: \mathbb{R} \rightarrow \mathbb{R}$ such that

$$
|b(x, t, s, \xi)| \leqslant \beta(s)|\xi|^{2}
$$

for almost all $(x, t) \in Q_{T}$ and all $(s, \xi) \in \mathbb{R} \times \mathbb{R}^{N}$.

As before, we will sometimes write $b(s, \xi)$ instead of $b(x, t, s, \xi)$. The two functions a and $b$ will not be independent from each other. In order to give the assumption on their connection, we define some auxiliary functions by

$$
\begin{aligned}
& \gamma(s)=\int_{0}^{s} \frac{\beta(\sigma)}{\alpha(\sigma)} \mathrm{d} \sigma, \\
& \Psi(s)=\int_{0}^{s} \alpha(\sigma) \mathrm{e}^{|\gamma(\sigma)|} \mathrm{d} \sigma, \\
& \Phi(s)=\int_{0}^{s} \Psi(\sigma) \mathrm{e}^{|\gamma(\sigma)|} \mathrm{d} \sigma .
\end{aligned}
$$

\section{Relation between a and $b$.}

(C1) We assume that there exists a constant $C_{1}>0$ such that

$$
\mathrm{e}^{|\gamma(s)|} \leqslant C_{1}(1+|\Psi(s)|)
$$

for all $s \in \mathbb{R}$.

Remark 2.1. It is easy to see that condition (C1) includes, for example, the case where

$$
\beta=\beta_{1}+\beta_{2},
$$

with

$$
\frac{\beta_{1}}{\alpha} \in L^{1}(\mathbb{R}), \quad \frac{\beta_{2}}{\alpha^{2}} \in L^{\infty}(\mathbb{R}),
$$

but is strictly more general, as we can see in the following counterexample.

Example 2.1. Consider two functions defined by $\alpha(s)=1$ and

$$
\beta(s)= \begin{cases}0, & \text { if } s \leqslant 1 ; \\ n \pi|\sin (n \pi s)|, & \text { if } s \in\left[n, n+\frac{1}{n}\right], n=1,2, \ldots ; \\ 0, & \text { if } s \in\left[n+\frac{1}{n}, n+1\right], n=1,2, \ldots\end{cases}
$$

Obviously both functions are continuous and by elementary arguments the following facts can be proved:

(1) $\int_{n}^{n+1} \beta(s) \mathrm{d} s=2$ for every $n=1,2, \ldots$, so that $\gamma(n)=2(n-1)$.

(2) $2 s-4 \leqslant \gamma(s) \leqslant 2 s$ for all $s \geqslant 0$.

(3) $\frac{\mathrm{e}^{-4}}{2}\left(\mathrm{e}^{2 s}-1\right) \leqslant \Psi(s)$ for all $s \geqslant 0$.

(4) $\mathrm{e}^{\gamma(s)} \leqslant \mathrm{e}^{2 s} \leqslant\left(\mathrm{e}^{2 s}-1\right)+2 \mathrm{e}^{4} \leqslant 2 \mathrm{e}^{4}(1+\Psi(s))$ for all $s \geqslant 0$.

(5) $\beta \notin L^{1}(\mathbb{R})+L^{\infty}(\mathbb{R})$. 
From the last two points, we may conclude that condition (C1) is strictly stronger than the one stated in the previous remark.

Remark 2.2. Let us observe that, on account of (C1),

$$
\alpha \notin L^{1}(0,+\infty) \cup L^{1}(-\infty, 0) \quad \Longleftrightarrow \quad \alpha \mathrm{e}^{|\gamma|} \notin L^{1}(0,+\infty) \cup L^{1}(-\infty, 0) .
$$

Indeed, on the one hand,

$$
\left|\int_{0}^{s} \alpha(\sigma) \mathrm{d} \sigma\right| \leqslant\left|\int_{0}^{s} \alpha(\sigma) \mathrm{e}^{|\gamma(\sigma)|} \mathrm{d} \sigma\right|
$$

and so $\alpha \notin L^{1}(0,+\infty) \cup L^{1}(-\infty, 0)$ implies $\alpha \mathrm{e}^{|\gamma|} \notin L^{1}(0,+\infty) \cup L^{1}(-\infty, 0)$.

On the other hand, it follows from (C1) that

$$
\frac{\Psi^{\prime}(s)}{1+|\Psi(s)|}=\frac{\alpha(s) \mathrm{e}^{|\gamma(s)|}}{1+|\Psi(s)|} \leqslant C_{1} \alpha(s) .
$$

Consequently,

$$
\log (1+|\Psi(s)|) \leqslant C_{1}\left|\int_{0}^{s} \alpha(\sigma) \mathrm{d} \sigma\right| \Longrightarrow|\Psi(s)| \leqslant \mathrm{e}^{C_{1}\left|\int_{0}^{s} \alpha(\sigma) \mathrm{d} \sigma\right|} .
$$

Hence, $\alpha \mathrm{e}^{|\gamma|} \notin L^{1}(0,+\infty) \cup L^{1}(-\infty, 0)$ implies $\alpha \notin L^{1}(0,+\infty) \cup L^{1}(-\infty, 0)$ and the two conditions are equivalent. Let us finally observe that both are equivalent to

$$
\lim _{s \rightarrow \pm \infty} \Psi(s)= \pm \infty
$$

As a consequence, it yields that the function $\Phi$ is superlinear at infinity, that is,

$$
\lim _{s \rightarrow \pm \infty} \frac{\Phi(s)}{|s|}=+\infty
$$

Assumptions on the data. We require that

$$
\int_{\Omega} \Phi\left(u_{0}(x)\right) \mathrm{d} x<\infty
$$

and that

$$
f(x, t) \in L^{r}\left(0, T ; L^{q}(\Omega)\right),
$$

with

$$
1<r<+\infty, \quad \frac{N}{2}<q<+\infty \quad \text { and } \quad \frac{1}{r}+\frac{N}{2 q}=1 .
$$

When the last equality is satisfied, we say that the couple $(r, q)$ belongs to the so called Aronson-Serrin curve, beyond which, in the classical case $\beta=0$ and $\alpha(s) \geqslant \alpha_{0}>0$ for every $s \in \mathbb{R}$, solutions are bounded (see [3]).

The main existence result will be the following. We will always assume that (A1), (A2), (B1) and (C1) are satisfied. 
Theorem 2.1. If (15) and (16) hold, then there exists a function $u \in C\left([0, T] ; L^{1}(\Omega)\right)$ which is a distributional solution of problem (6), satisfying

$$
\begin{aligned}
& \mathbf{a}(x, t, u) \nabla u \in L^{2}\left(Q_{T}\right), \quad b(x, t, u, \nabla u) \in L^{1}\left(Q_{T}\right), \\
& \Psi(u) \in L^{2}\left(0, T ; H_{0}^{1}(\Omega)\right) \cap L^{\infty}\left(0, T ; L^{2}(\Omega)\right), \\
& \sup _{\tau \in[0, T]} \int_{\Omega} \Phi(u(x, \tau))<+\infty,
\end{aligned}
$$

where $\Psi$ and $\Phi$ are defined in (12) and (13), respectively. Moreover, if the initial datum $u_{0}$ satisfies the stronger assumption

$$
\int_{\Omega} \Phi^{(\delta)}\left(u_{0}\right)<\infty,
$$

for some $\delta>0$, where

$$
\Phi^{(\delta)}(s)=\int_{0}^{s}|\Psi(\sigma)|^{2 \delta} \Psi(\sigma) \mathrm{e}^{|\gamma(\sigma)|} \mathrm{d} \sigma,
$$

then (17) holds with $\Psi(u)$ replaced by $|\Psi(u)|^{\delta} \Psi(u)$, while (18) holds with $\Phi(u)$ replaced by $\Phi^{(\delta)}(u)$.

Remark 2.3. It is worth simplifying our situation to the following (more classical) model problem:

$$
\begin{cases}u_{t}-\Delta u=|\nabla u|^{2}+f, & \text { in } Q_{T} ; \\ u(x, t)=0, & \text { on } \Sigma_{T} ; \\ u(x, 0)=u_{0}, & \text { in } \Omega .\end{cases}
$$

Then our main result states that an initial datum satisfying $\int_{\Omega}\left(\mathrm{e}^{\left|u_{0}\right|}-1\right)^{2}<\infty$ and $f \in L^{r}\left(0, T ; L^{q}(\Omega)\right)$, with $(r, q)$ on the Aronson-Serrin curve, imply the existence of a distributional solution $u$ such that

$$
\mathrm{e}^{|u|}-1 \in L^{\infty}\left(0, T ; L^{2}(\Omega)\right) \cap L^{2}\left(0, T ; H_{0}^{1}(\Omega)\right) .
$$

Similar results in a more general setting can be found also in [13] and in [19].

Remark 2.4. One can check, by adapting the proof, that the result of Theorem 2.1 also holds true in the case where the datum $f$ satisfies a limit case in (16), i.e. $f \in L^{\infty}\left(0, T ; L^{N / 2}(\Omega)\right)$, provided the following condition is verified:

$$
\text { for every } \varepsilon>0 \text { there exist two functions } f_{1}^{(\varepsilon)}(x, t), f_{2}^{(\varepsilon)}(x, t) \text { such that }
$$

$$
f=f_{1}^{(\varepsilon)}+f_{2}^{(\varepsilon)}, \quad f_{1}^{(\varepsilon)} \in L^{\infty}\left(Q_{T}\right) \quad \text { and } \quad\left\|f_{2}^{(\varepsilon)}\right\|_{L^{\infty}\left(0, T ; L^{N / 2}(\Omega)\right)} \leqslant \varepsilon .
$$

This is true, for instance, if $f(x, t)=f(x) \in L^{N / 2}(\Omega)$ or if $f \in C\left([0, T] ; L^{N / 2}(\Omega)\right)$.

Remark 2.5. Assuming that the initial datum $u_{0}(x)$ is summable enough, we are interested in the best estimates for $u$, possibly replacing the function $\beta$ with a greater function $\beta^{*}$ which satisfies again condition (C1). For instance, if $\alpha(s) \equiv 1$ and $\beta(s)=1 /(1+|s|)$, it would be better to choose $\beta^{*}(s) \equiv 1 \geqslant \beta(s)$, which would provide better estimates on $u$. The function defined by

$$
\bar{\gamma}(s)=C \int_{0}^{s} \alpha(\sigma) \mathrm{d} \sigma+C^{\prime}
$$


realize the equality in condition $(\mathrm{C} 1)$. Therefore anytime that there exists $\beta^{*} \geqslant \beta$ such that the corresponding function $\gamma^{*}(s)=\int_{0}^{s} \frac{\beta^{*}(\sigma)}{\alpha(\sigma)} \mathrm{d} \sigma$ satisfies

$$
\bar{\gamma}(s)-C_{2} \leqslant \gamma^{*}(s) \leqslant \bar{\gamma}(s)+C_{3}
$$

for some positive constants $C_{2}, C_{3}$, we get the best estimate choosing $\gamma^{*}$ instead of $\gamma$ (which means $\beta^{*}$ instead of $\beta$ ). This is the case for $\beta$ as in Remark 2.1. Indeed, if

$$
\beta=\beta_{1}+\beta_{2},
$$

with

$$
\frac{\beta_{1}}{\alpha} \in L^{1}(\mathbb{R}), \quad \frac{\beta_{2}}{\alpha^{2}} \in L^{\infty}(\mathbb{R}),
$$

then

$$
\beta \leqslant \beta^{*}=\beta_{1}+C \alpha^{2}
$$

and condition (20) is satisfied. This is also the case for the oscillating function $\beta$ in the Example 2.1, as one can easily see. Therefore the function $\bar{\gamma}$ and the corresponding function $\bar{\Psi}$ play an essential role in the optimality of the estimates in all the known cases for $\beta$. Let us point out that condition (20) implies condition (C1), while condition (C1) implies, via Gronwall's lemma, the second inequality in condition (20).

Remark 2.6. Let us consider the case $\alpha=1$ and write condition (C1) as

$$
\mathrm{e}^{|\gamma(s)|} \leqslant C+C\left|\int_{0}^{s} \mathrm{e}^{|\gamma(\sigma)|} \mathrm{d} \sigma\right|
$$

and applying Gronwall's lemma, we get $\mathrm{e}^{|\gamma(s)|} \leqslant C \mathrm{e}^{C|s|}$, that is,

$$
|\gamma(s)| \leqslant C(|s|+1) \text {. }
$$

In some papers concerning parabolic problems with coercive operators $(\alpha \equiv 1)$ and quadratic terms (see, for instance, $[8,19,20]$ and [13]) authors assume that the function $\beta$ is bounded, which obviously implies (21). Thus, in the case $\alpha \equiv 1$, our condition does not allow a greater growth on function $\gamma$; however, we can consider also unbounded oscillating functions $\beta(s)$ like in Example 2.1.

It is worth remarking that our condition (C1) points out the role of function $\gamma$, instead of $\beta$, in obtaining existence of solutions.

It is also worth noting that the estimates given in the previous theorem could be very weak. Indeed, for example, we can have, for $s>0, \alpha(s)=1 /(s+\mathrm{e}) \log (s+\mathrm{e})$ and $\beta(s)=\alpha^{2}(s)$, which gives $\gamma(s)=\log (\log (s+\mathrm{e}))$ and $\Psi(s)=\log (s+\mathrm{e})-1$.

Finally, if the data are more regular, one can prove the existence of bounded solutions. More precisely, we assume that

$$
u_{0}(x) \in L^{\infty}(\Omega),
$$

and

$$
f(x, t) \in L^{r}\left(0, T ; L^{q}(\Omega)\right) \quad \text { with } \frac{1}{r}+\frac{N}{2 q}<1 .
$$

Theorem 2.2. If (22) and (23) hold, then the solution found by Theorem 2.1 is bounded. 


\section{Approximate problems. Some useful results}

To prove our result, first of all, we have to consider approximating problems. To guarantee coerciveness, we will change the function a defining

$$
\mathbf{a}_{n}(x, t, s)=\mathbf{a}(x, t, s)+\frac{1}{n} \mathbf{I},
$$

where $\mathbf{I}$ is the identity matrix, and will truncate the others terms of our equation. Consequently, we now define

$$
\alpha_{n}(s)=\alpha(s)+\frac{1}{n} \quad \text { and } \quad \beta_{n}(s)=\alpha_{n}(s) \frac{\beta(s)}{\alpha(s)},
$$

so that $\beta_{n} \geqslant \beta$ and $\beta_{n} / \alpha_{n}=\beta / \alpha$ for all $n \in \mathbb{N}$. It also yields

$$
\left(\mathbf{a}_{n}(x, t, s) \xi, \xi\right) \geqslant \alpha_{n}(s)|\xi|^{2}, \quad \text { and } \quad\left|T_{n}(b(x, t, s, \xi))\right| \leqslant \beta_{n}(s)|\xi|^{2} .
$$

Let us next define the functions

$$
\begin{aligned}
& \Psi_{n}(s)=\int_{0}^{s} \alpha_{n}(\sigma) \mathrm{e}^{|\gamma(\sigma)|} \mathrm{d} \sigma, \quad \Phi_{n}(s)=\int_{0}^{s} \Psi_{n}(\sigma) \mathrm{e}^{|\gamma(\sigma)|} \mathrm{d} \sigma, \\
& \Phi_{n}^{(\delta)}(s)=\int_{0}^{s}\left|\Psi_{n}(\sigma)\right|^{2 \delta} \Psi_{n}(\sigma) \mathrm{e}^{|\gamma(\sigma)|} \mathrm{d} \sigma, \quad \text { where } \delta>0,
\end{aligned}
$$

and

$$
h(s)=\int_{0}^{s} \mathrm{e}^{|\gamma(\sigma)|} \mathrm{d} \sigma .
$$

Observe that it follows

$$
|h(s)| \geqslant|s|, \quad \Psi_{n}(s)=\Psi(s)+\frac{1}{n} h(s) \quad \text { and } \quad \Phi_{n}(s)=\Phi(s)+\frac{1}{2 n} h(s)^{2}
$$

and so

$$
\left|\Psi_{n}(s)\right| \geqslant|\Psi(s)| \text { and } \Phi_{n}(s) \geqslant \Phi(s) \geqslant 0 \quad \text { for all } s \in \mathbb{R}
$$

On the other hand, we need to regularize our initial datum. We will take an approximating sequence whose properties are stated in the following proposition:

Proposition 3.1. If (15) holds, there exists a sequence $\left\{u_{0, n}\right\}$ in $L^{\infty}(\Omega) \cap H_{0}^{1}(\Omega)$ such that

$$
\begin{aligned}
& \frac{1}{n}\left\|u_{0, n}\right\|_{H_{0}^{1}(\Omega)} \rightarrow 0 \quad \text { as } n \rightarrow \infty, \\
& \Phi\left(u_{0, n}\right) \rightarrow \Phi\left(u_{0}\right) \quad \text { a.e. and strongly in } L^{1}(\Omega), \\
& \Phi_{n}\left(u_{0, n}\right) \rightarrow \Phi\left(u_{0}\right) \quad \text { a.e. and strongly in } L^{1}(\Omega) .
\end{aligned}
$$

In the case where the stronger assumption (19) is satisfied, one may assume that $\Phi_{n}^{(\delta)}\left(u_{0, n}\right)$ is also uniformly bounded. Finally, if $u_{0}$ is bounded, one may assume that $u_{0, n}$ are also uniformly bounded.

Proof. Let $\left\{\ell_{n}\right\}_{n}$ be a strictly increasing sequence of positive numbers satisfying $\lim _{n \rightarrow \infty} \ell_{n}=+\infty$ and

$$
\max \left\{h\left(\ell_{n}\right),-h\left(-\ell_{n}\right)\right\} \leqslant \sqrt[4]{n} .
$$


Furthermore, consider a sequence $\left\{v_{0, n}\right\}_{n}$ such that $v_{0, n} \in L^{\infty}(\Omega) \cap H_{0}^{1}(\Omega)$,

$$
\frac{1}{n}\left\|v_{0, n}\right\|_{H_{0}^{1}(\Omega)} \rightarrow 0 \quad \text { and } \quad \Phi\left(v_{0, n}\right) \rightarrow \Phi\left(u_{0}\right) \quad \text { a.e. and strongly in } L^{1}(\Omega)
$$

We finally denote $u_{0, n}=T_{\ell_{n}} v_{0, n}$; obviously it satisfies $u_{0, n} \in L^{\infty}(\Omega) \cap H_{0}^{1}(\Omega)$ and $\left\|u_{0, n}\right\|_{H_{0}^{1}(\Omega)} \leqslant\left\|v_{0, n}\right\|_{H_{0}^{1}(\Omega)}$; thus, (30) is proved. Moreover, since

$$
\int_{\Omega}\left|\Phi\left(v_{0, n}\right)-\Phi\left(u_{0, n}\right)\right| \leqslant \int_{\left\{\left|v_{0, n}\right| \geqslant \ell_{n}\right\}} \Phi\left(v_{0, n}\right) \rightarrow 0,
$$

we deduce that (31) holds. From here and the inequalities $\Phi\left(u_{0, n}\right) \leqslant \Phi_{n}\left(u_{0, n}\right) \leqslant \Phi_{n}\left(v_{0, n}\right) \leqslant \Phi\left(v_{0, n}\right)+\frac{1}{2 \sqrt{n}}$ (see (33)), we obtain (32). The proof of the final statements is trivial.

Let us consider the approximating problems

$$
\begin{cases}\left(u_{n}\right)_{t}-\operatorname{div}\left(\mathbf{a}_{n}\left(x, t, u_{n}\right) \nabla u_{n}\right)=T_{n} b\left(x, t, u_{n}, \nabla u_{n}\right)+T_{n} f, & \text { in } Q_{T} \\ u_{n}(x, t)=0, & \text { on } \Sigma_{T} \\ u_{n}(x, 0)=u_{0, n}(x), & \text { in } \Omega .\end{cases}
$$

It is quite classical (see, for instance, [21]) that problem (34) admits at least one weak solution $u_{n} \in L^{\infty}\left(Q_{T}\right) \cap$ $L^{2}\left(0, T ; H_{0}^{1}(\Omega)\right) \cap C\left([0, T] ; L^{2}(\Omega)\right)$.

In order to prove first a priori estimates on our approximate solutions $u_{n}$ and then the convergence of the sequence $\left\{u_{n}\right\}_{n}$, we need the following cancellation result, which is a variant of that proved in [9], Lemma 2.1.

Proposition 3.2. Assume that $u_{n}$ is a bounded weak solution of (34).

(1) If $v \in L^{\infty}\left(Q_{T}\right) \cap L^{2}\left(0, T ; H_{0}^{1}(\Omega)\right)$, then

$$
\int_{0}^{t}\left\langle\left(u_{n}\right)_{t}, \mathrm{e}^{\operatorname{sign}(v) \gamma\left(u_{n}\right)} v\right\rangle+\int_{0}^{t} \int_{\Omega} \mathrm{e}^{\operatorname{sign}(v) \gamma\left(u_{n}\right)} \mathbf{a}_{n}\left(x, t, u_{n}\right) \nabla u_{n} \nabla v \leqslant \int_{0}^{t} \int_{\Omega} \mathrm{e}^{\operatorname{sign}(v) \gamma\left(u_{n}\right)} v T_{n}(f)
$$

holds for all $t \in[0, T]$, where $\langle\cdot, \cdot\rangle$ denotes the duality pairing between the spaces $H^{-1}(\Omega)$ and $H_{0}^{1}(\Omega)$.

(2) If $\psi$ is a locally Lipschitz continuous and increasing function such that $\psi(0)=0$, then

$$
\sup _{\tau \in[0, T]} \int_{\Omega} \phi\left(u_{n}(\tau)\right)+\int_{Q_{T}} \alpha_{n}\left(u_{n}\right) \mathrm{e}^{\left|\gamma\left(u_{n}\right)\right|} \psi^{\prime}\left(u_{n}\right)\left|\nabla u_{n}\right|^{2} \leqslant \int_{Q_{T}}|f| \mathrm{e}^{\left|\gamma\left(u_{n}\right)\right|}\left|\psi\left(u_{n}\right)\right|+\int_{\Omega} \phi\left(u_{0, n}\right) ;
$$

where $\phi(s)=\int_{0}^{s} \mathrm{e}^{|\gamma(\sigma)|} \psi(\sigma) \mathrm{d} \sigma$.

Another important tool we will use to get a priori estimates is the well known Gagliardo-Nirenberg's inequality for evolution spaces (see [15]):

Lemma 3.1. Let $\Omega$ be a bounded open set of $\mathbb{R}^{N}$ and $T$ be a real positive number. Let $v(x, t)$ be a function such that

$$
v \in L^{\infty}\left(0, T ; L^{2}(\Omega)\right) \cap L^{2}\left(0, T ; H_{0}^{1}(\Omega)\right) .
$$

Then $v \in L^{\rho}\left(0, T ; L^{\sigma}(\Omega)\right)$, where

$$
2 \leqslant \sigma \leqslant \frac{2 N}{N-2}, \quad 2 \leqslant \rho \leqslant \infty
$$


and

$$
\frac{N}{\sigma}+\frac{2}{\rho}=\frac{N}{2}
$$

and the following estimate holds

$$
\int_{0}^{T}\|v(t)\|_{L^{\sigma}(\Omega)}^{\rho} \mathrm{d} t \leqslant C(N)\|v\|_{L^{\infty}\left(0, T ; L^{2}(\Omega)\right)}^{\rho-2} \int_{0}^{T}\|\nabla v(t)\|_{L^{2}\left(\Omega ; \mathbb{R}^{N}\right)}^{2} \mathrm{~d} t .
$$

\section{A priori estimate on Aronson-Serrin's curve: unbounded solutions}

In this section, we will obtain a priori estimates under the assumptions (15) and (16).

Proposition 4.1. Assume that (15) and (16) are satisfied, and let $\left\{u_{n}\right\}_{n}$ be a sequence of solutions of problems (34). Then there exists a constant $C>0$, depending only on the data of problem (6), such that, for every $n \in \mathbb{N}$,

$$
\begin{aligned}
& \int_{\Omega} \Phi_{n}\left(u_{n}(x, \tau)\right) \leqslant C \quad \text { for almost all } \tau \in[0, T], \\
& \int_{\Omega} \Psi_{n}^{2}\left(u_{n}(x, \tau)\right) \leqslant C \quad \text { for almost all } \tau \in[0, T], \\
& \int_{Q_{T}}\left|\nabla \Psi_{n}\left(u_{n}\right)\right|^{2} \leqslant C,
\end{aligned}
$$

where $\Phi_{n}$ and $\Psi_{n}$ are defined by (26). Moreover, if $u_{0}$ satisfies (19) for some $\delta>0$, then there exists a constant $C_{\delta}>0$, depending only on $\delta$ and on the data of problem (6), such that, for every $n \in \mathbb{N}$,

$$
\begin{aligned}
& \int_{\Omega} \Phi_{n}^{(\delta)}\left(u_{n}(x, \tau)\right) \leqslant C_{\delta} \quad \text { for almost all } \tau \in[0, T], \\
& \int_{\Omega}\left|\Psi_{n}\left(u_{n}(x, \tau)\right)\right|^{2(\delta+1)} \leqslant C_{\delta} \quad \text { for almost all } \tau \in[0, T], \\
& \int_{Q_{T}}\left|\nabla\left(\left|\Psi_{n}\left(u_{n}\right)\right|^{\delta+1}\right)\right|^{2} \leqslant C_{\delta},
\end{aligned}
$$

where $\Phi_{n}^{(\delta)}$ is defined by (27).

Proof. We take $\psi=\Psi_{n}$ in part (2) of Proposition 3.2, getting

$$
\sup _{\tau \in[0, T]} \int_{\Omega} \Phi_{n}\left(u_{n}(x, \tau)\right)+\int_{Q_{T}} \alpha_{n}\left(u_{n}\right) \mathrm{e}^{\left|\gamma\left(u_{n}\right)\right|} \Psi_{n}^{\prime}\left(u_{n}\right)\left|\nabla u_{n}\right|^{2} \leqslant \int_{Q_{T}}|f| \mathrm{e}^{\left|\gamma\left(u_{n}\right)\right|} \Psi_{n}\left(u_{n}\right)+C,
$$

where $C$ is a constant such that

$$
\int_{\Omega} \Phi_{n}\left(u_{0, n}\right) \leqslant C
$$


(see (32)). Let us point out that, by the definition of function $\Psi_{n}$,

$$
\alpha_{n}\left(u_{n}\right) \mathrm{e}^{\left|\gamma\left(u_{n}\right)\right|} \Psi_{n}^{\prime}\left(u_{n}\right)\left|\nabla u_{n}\right|^{2}=\left|\nabla \Psi_{n}\left(u_{n}\right)\right|^{2} .
$$

Moreover hypothesis (C1) implies the following estimates on the right-hand side (recall that $|\Psi(s)| \leqslant\left|\Psi_{n}(s)\right|$ for every $s \in \mathbb{R}$ )

$$
\begin{aligned}
\int_{Q_{T}}|f| \mathrm{e}^{\left|\gamma\left(u_{n}\right)\right|}\left|\Psi_{n}\left(u_{n}\right)\right| & \leqslant C \int_{Q_{T}}|f|\left|\Psi_{n}\left(u_{n}\right)\right|\left(1+\left|\Psi_{n}\left(u_{n}\right)\right|\right) \leqslant C\left(\frac{3}{2} \int_{Q_{T}}|f| \Psi_{n}^{2}\left(u_{n}\right)+\frac{1}{2} \int_{Q_{T}}|f|\right) \\
& =C \int_{Q_{T}}|f| \Psi_{n}^{2}\left(u_{n}\right)+C .
\end{aligned}
$$

From the previous estimates we get

$$
\sup _{\tau \in[0, T]} \int_{\Omega} \Phi_{n}\left(u_{n}(x, \tau)\right)+\int_{Q_{T}}\left|\nabla \Psi_{n}\left(u_{n}\right)\right|^{2} \leqslant C \int_{Q_{T}}|f| \Psi_{n}^{2}\left(u_{n}\right)+C .
$$

Let us now estimate the term $\int_{Q_{T}}|f| \Psi_{n}^{2}\left(u_{n}\right)$ in the right-hand side of inequality (44). Having in mind hypothesis (16) on $f$, and applying Hölder's inequality, we obtain

$$
\int_{Q_{T}}|f| \Psi_{n}^{2}\left(u_{n}\right) \leqslant\|f\|_{r, q}\left\|\Psi_{n}^{2}\left(u_{n}\right)\right\|_{r^{\prime}, q^{\prime}}=\|f\|_{r, q}\left\|\Psi_{n}\left(u_{n}\right)\right\|_{2 r^{\prime}, 2 q^{\prime}}^{2} .
$$

Let us define $\rho=2 r^{\prime}, \sigma=2 q^{\prime}$, and point out that $(\rho, \sigma)$ satisfy conditions (36) and (37) of the GagliardoNirenberg Lemma 3.1, and therefore

$$
\begin{aligned}
\left\|\Psi_{n}\left(u_{n}\right)\right\|_{2 r^{\prime}, 2 q^{\prime}}^{2} & \leqslant C\left\|\Psi_{n}\left(u_{n}\right)\right\|_{\infty, 2}^{2 / r}\left[\int_{Q_{T}}\left|\nabla \Psi_{n}\left(u_{n}\right)\right|^{2}\right]^{1 / r^{\prime}} \\
& \leqslant C\left[\sup _{\tau \in[0, T]} \int_{\Omega} \Phi_{n}\left(u_{n}(x, \tau)\right)\right]^{1 / r}\left[\int_{Q_{T}}\left|\nabla \Psi_{n}\left(u_{n}\right)\right|^{2}\right]^{1 / r^{\prime}},
\end{aligned}
$$

where we have used the inequality

$$
\Phi_{n}(s) \geqslant \frac{1}{\|\alpha+1\|_{\infty}} \int_{0}^{s} \alpha_{n}(\sigma) \mathrm{e}^{|\gamma(\sigma)|} \Psi_{n}(\sigma) \mathrm{d} \sigma=\frac{\Psi_{n}^{2}(s)}{2\|\alpha+1\|_{\infty}} .
$$

Using (44)-(46) and applying Young's inequality, we get

$$
\begin{aligned}
\sup _{\tau \in[0, T]} \int_{\Omega} \Phi_{n}\left(u_{n}(x, \tau)\right)+\int_{Q_{T}}\left|\nabla \Psi_{n}\left(u_{n}\right)\right|^{2} & \leqslant C\|f\|_{r, q}\left[\sup _{\tau \in[0, T]} \int_{\Omega} \Phi_{n}\left(u_{n}(x, \tau)\right)\right]^{1 / r}\left[\int_{Q_{T}}\left|\nabla \Psi_{n}\left(u_{n}\right)\right|^{2}\right]^{1 / r^{\prime}}+C \\
& \leqslant \frac{1}{2} \int_{Q_{T}}\left|\nabla \Psi_{n}\left(u_{n}\right)\right|^{2}+C\|f\|_{r, q}^{r} \sup _{\tau \in[0, T]} \int_{\Omega} \Phi_{n}\left(u_{n}(x, \tau)\right)+C .
\end{aligned}
$$

If $\|f\|_{r, q}$ is sufficiently small we get the desired estimates on

$$
\sup _{\tau \in[0, T]} \int_{\Omega} \Phi_{n}\left(u_{n}(x, \tau)\right)+\int_{Q_{T}}\left|\nabla \Psi_{n}\left(u_{n}\right)\right|^{2},
$$


and estimate (39) follows from (47). If this is not the case, let us take $t_{1}$ instead of $T$ in such a way that

$$
C\|f\|_{L^{r}\left(0, t_{1} ; L^{q}(\Omega)\right)}^{r}=\frac{1}{2}
$$

By the previous argument we get estimate on

$$
\sup _{\tau \in\left[0, t_{1}\right]} \int_{\Omega} \Phi_{n}\left(u_{n}(x, \tau)\right)+\int_{Q_{t_{1}}}\left|\nabla \Psi_{n}\left(u_{n}\right)\right|^{2} .
$$

Then we take $t_{2}>t_{1}$ such that

$$
C\|f\|_{L^{r}\left(t_{1}, t_{2} ; L^{q}(\Omega)\right)}^{r}=\frac{1}{2}
$$

and we repeat the same argument as before.

It is clear that in a finite number of steps one covers the whole interval $[0, T]$, getting the quoted estimates.

In the case where $u_{0}$ satisfies the stronger assumption (19), we can assume that $\Phi_{n}^{(\delta)}\left(u_{0, n}\right)$ are also uniformly bounded in $L^{1}(\Omega)$; by taking $\psi=\left|\Psi_{n}\right|^{2 \delta} \Psi_{n}$ in part (2) of Proposition 3.2, one obtains:

$$
\sup _{\tau \in[0, T]} \int_{\Omega} \Phi_{n}^{(\delta)}\left(u_{n}(x, \tau)\right)+\int_{Q_{T}}\left|\nabla\left(\left|\Psi_{n}\left(u_{n}\right)\right|^{\delta+1}\right)\right|^{2} \leqslant C \int_{Q_{T}}|f|\left|\Psi_{n}\left(u_{n}\right)\right|^{2(\delta+1)}+C .
$$

It is easy to check that the function $\Phi_{n}^{(\delta)}$, defined by (27), satisfies the inequality

$$
\Phi_{n}^{(\delta)}(s) \geqslant \frac{\left|\Psi_{n}(s)\right|^{2(\delta+1)}}{2(\delta+1)\|\alpha+1\|_{\infty}}
$$

for every $s \in \mathbb{R}$. From (48) and (49) one easily obtains the estimates (41)-(43).

Corollary 4.1. The sequence $\left\{\mathbf{a}_{n}\left(x, t, u_{n}\right) \nabla u_{n}\right\}_{n}$ is bounded in $L^{2}\left(Q_{T} ; \mathbb{R}^{N}\right)$.

Proof. This is a straightforward consequence of (9) and (40). Indeed,

$$
\left|\mathbf{a}_{n}\left(x, t, u_{n}\right) \nabla u_{n}\right|^{2} \leqslant C_{0} \alpha_{n}\left(u_{n}\right)^{2}\left|\nabla u_{n}\right|^{2} \leqslant C_{0}\left[\alpha_{n}\left(u_{n}\right) \mathrm{e}^{\left|\gamma\left(u_{n}\right)\right|}\right]^{2}\left|\nabla u_{n}\right|^{2}=C_{0}\left|\nabla \Psi\left(u_{n}\right)\right|^{2} .
$$

Next, we will prove the estimates we need on the lower order term.

Proposition 4.2. The following statements hold true:

(1) There exist positive constants $C$ and $s_{0}$ such that

$$
\int_{\left\{\left|u_{n}\right|>k\right\}}\left|T_{n}\left(b\left(x, t, u_{n}, \nabla u_{n}\right)\right)\right| \leqslant C\left\|f \chi_{\left\{\left|u_{n}\right|>k\right\}}\right\|_{L^{r}\left(0, T ; L^{q}(\Omega)\right)}+C \int_{\Omega \cap\left\{\left|u_{0, n}\right|>k\right\}} \Phi\left(u_{0, n}\right)
$$

holds for every $n \in \mathbb{N}$ and $k \geqslant s_{0}$.

(2) The sequence $\left\{T_{n}\left(b\left(x, t, u_{n}, \nabla u_{n}\right)\right)\right\}_{n}$ is bounded in $L^{1}\left(Q_{T}\right)$.

Proof. On account of (25), the first claim of Proposition 4.2 is a straightforward consequence of the following inequality

$$
\int_{\left\{\left|u_{n}\right|>k\right\}} \beta_{n}\left(u_{n}\right)\left|\nabla u_{n}\right|^{2} \leqslant C\left\|f \chi_{\left\{\left|u_{n}\right|>k\right\}}\right\|_{r, q}+C \int_{\Omega \cap\left\{\left|u_{0, n}\right|>k\right\}} \Phi\left(u_{0, n}\right) .
$$


To see this, we begin by taking

$$
\psi(s)=\chi_{\{|s|>k\}}(s) \int_{k \operatorname{sign}(s)}^{s} \frac{\beta_{n}(\sigma)}{\alpha_{n}(\sigma)} \mathrm{e}^{-|\gamma(\sigma)|} \mathrm{d} \sigma=\chi_{\{|s|>k\}}(s) \operatorname{sign}(s)\left(\mathrm{e}^{-|\gamma(k \operatorname{sign}(s))|}-\mathrm{e}^{-|\gamma(s)|}\right)
$$

in (35). Dropping nonnegative terms, we deduce that

$$
\int_{\left\{\left|u_{n}\right|>k\right\}} \beta_{n}\left(u_{n}\right)\left|\nabla u_{n}\right|^{2} \leqslant \int_{\left\{\left|u_{n}\right|>k\right\}}|f|\left(\mathrm{e}^{\left|\gamma\left(u_{n}\right)\right|-\left|\gamma\left(k \operatorname{sign}\left(u_{n}\right)\right)\right|}-1\right)+\int_{\Omega} \phi_{k}\left(u_{0, n}\right),
$$

where

$$
\phi_{k}(s)=\chi_{\{|s|>k\}}(s) \operatorname{sign}(s) \int_{k \operatorname{sign}(s)}^{s}\left(\mathrm{e}^{|\gamma(\sigma)|-|\gamma(k \operatorname{sign}(\sigma))|}-1\right) \mathrm{d} \sigma .
$$

Since $\lim _{s \rightarrow \pm \infty} \Psi(s)= \pm \infty$, we may find $s_{0}>0$ such that $|s| \geqslant s_{0}$ implies $|\Psi(s)| \geqslant 1$. So that, if $k \geqslant s_{0}$, then

$$
\phi_{k}(s) \leqslant \chi_{\{|s|>k\}}(s) \operatorname{sign}(s) \int_{k \operatorname{sign}(s)}^{s} \mathrm{e}^{|\gamma(\sigma)|} \mathrm{d} \sigma \leqslant \chi_{\{|s|>k\}}(s) \int_{k \operatorname{sign}(s)}^{s} \Psi(\sigma) \mathrm{e}^{|\gamma(\sigma)|} \mathrm{d} \sigma \leqslant \Phi(s) \chi_{\{|s|>k\}}(s) .
$$

On the other hand, if $k \geqslant s_{0}$,

$$
\int_{\left\{\left|u_{n}\right|>k\right\}}|f|\left(\mathrm{e}^{\left|\gamma\left(u_{n}\right)\right|-\left|\gamma\left(k \operatorname{sign}\left(u_{n}\right)\right)\right|}-1\right) \leqslant \int_{\left\{\left|u_{n}\right|>k\right\}}|f| \mathrm{e}^{\left|\gamma\left(u_{n}\right)\right|}\left|\Psi_{n}\left(u_{n}\right)\right| \leqslant C \int_{\left\{\left|u_{n}\right|>k\right\}}|f|\left|\Psi_{n}\left(u_{n}\right)\right|^{2}+C \int_{\left\{\left|u_{n}\right|>k\right\}}|f|
$$

as a consequence of (C1) and Young's inequality. Thus, applying Hölder's inequality, we obtain

$$
\int_{\left\{\left|u_{n}\right|>k\right\}}|f|\left(\mathrm{e}^{\left|\gamma\left(u_{n}\right)\right|-\left|\gamma\left(k \operatorname{sign}\left(u_{n}\right)\right)\right|}-1\right) \leqslant C\left\|f \chi_{\left\{\left|u_{n}\right|>k\right\}}\right\|_{r, q}\left\|\Psi_{n}\left(u_{n}\right)\right\|_{2 r^{\prime}, 2 q^{\prime}}^{2}+C\left\|f \chi_{\left\{\left|u_{n}\right|>k\right\}}\right\|_{r, q} .
$$

Since the sequence $\left\{\left\|\Psi\left(u_{n}\right)\right\|_{2 r^{\prime}, 2 q^{\prime}}\right\}$ is bounded (by (39), (40) and Lemma 3.1), it follows that

$$
\int_{\left\{\left|u_{n}\right|>k\right\}}|f|\left(\mathrm{e}^{\left|\gamma\left(u_{n}\right)\right|-\left|\gamma\left(k \operatorname{sign}\left(u_{n}\right)\right)\right|}-1\right) \leqslant C\left\|f \chi_{\left\{\left|u_{n}\right|>k\right\}}\right\|_{r, q}
$$

from where the first assertion of Proposition 4.2 follows.

The second claim of Proposition 4.2 is proved by taking

$$
\psi(s)=\int_{0}^{s} \frac{\beta_{n}(\sigma)}{\alpha_{n}(\sigma)} \mathrm{e}^{-|\gamma(\sigma)|} \mathrm{d} \sigma=\operatorname{sign}(s)\left(1-\mathrm{e}^{-|\gamma(s)|}\right)
$$

in (35). Indeed, then

$$
\int_{Q_{T}} \beta_{n}\left(u_{n}\right)\left|\nabla u_{n}\right|^{2} \leqslant \int_{Q_{T}}|f|\left(\mathrm{e}^{\left|\gamma\left(u_{n}\right)\right|}-1\right)+\int_{\Omega} \phi\left(u_{0, n}\right),
$$

where $\phi(s)=\operatorname{sign}(s) \int_{0}^{s}\left(\mathrm{e}^{|\gamma(\sigma)|}-1\right) \mathrm{d} \sigma$. As above, we obtain

$$
\begin{aligned}
\int_{Q_{T}}|f|\left(\mathrm{e}^{\left|\gamma\left(u_{n}\right)\right|}-1\right) & =\int_{\substack{\left\{\left|u_{n}\right| \leqslant s_{0}\right\}\\
}}|f|\left(\mathrm{e}^{\left|\gamma\left(u_{n}\right)\right|}-1\right)+\int_{\left\{\left|u_{n}\right|>s_{0}\right\}}|f|\left(\mathrm{e}^{\left|\gamma\left(u_{n}\right)\right|}-1\right) \\
& \leqslant C\|f\|_{L^{1}\left(Q_{T}\right)}+C\left\|f \chi_{\left\{\left|u_{n}\right|>s_{0}\right\}}\right\|_{r, q} .
\end{aligned}
$$


On the other hand,

$$
\phi(s)=\operatorname{sign}(s) \int_{0}^{s_{0} \operatorname{sign}(s)}\left(\mathrm{e}^{|\gamma(\sigma)|}-1\right) \mathrm{d} \sigma+\operatorname{sign}(s) \int_{s_{0} \operatorname{sign}(s)}^{s}\left(\mathrm{e}^{|\gamma(\sigma)|}-1\right) \mathrm{d} \sigma \leqslant C+\Phi(s) \chi_{\left\{|s| \geqslant s_{0}\right\}}
$$

and so

$$
\int_{\Omega} \phi\left(u_{0, n}\right) \leqslant C+\int_{\left\{\left|u_{0, n}\right| \geqslant s_{0}\right\}} \Phi\left(u_{0, n}\right) .
$$

Hence, from these inequalities, having in mind (50) and (31), we conclude that the sequence $\left\{\beta_{n}\left(u_{n}\right)\left|\nabla u_{n}\right|^{2}\right\}_{n}$ is bounded in $L^{1}\left(Q_{T}\right)$. The boundedness of $\left\{T_{n}\left(b\left(u_{n}, \nabla u_{n}\right)\right)\right\}_{n}$ then follows from (25).

Taking into account that $u_{n}$ is a solution of problem (34), the two previous results imply the following consequence.

Corollary 4.2. The sequence $\left\{\left(u_{n}\right)_{t}\right\}_{n}$ is bounded in $L^{2}\left(0, T ; H^{-1}(\Omega)\right)+L^{1}\left(Q_{T}\right)$.

\section{Beyond Aronson-Serrin's curve: bounded solutions}

In this section, we will prove Theorem 2.2. Actually, we only have to prove an $L^{\infty}$-estimate for $\left\{u_{n}\right\}$, since after that Theorem 2.2 is easy to see following the reasoning of ([9], Theorem 1.1). The estimate is as follows.

Theorem 5.1. If $1 / r+N / 2 q<1, u_{0, n}$ are bounded in $L^{\infty}(\Omega)$ and $u_{n} \in L^{2}\left(0, T ; H_{0}^{1}(\Omega)\right)$ is a distributional solution of (34), then there exists a constant $C>0$, depending only on the parameters of the problem, such that

$$
\left\|\Psi_{n}\left(u_{n}\right)\right\|_{\infty} \leqslant C
$$

which implies, taking (14) and (29) into account,

$$
\left\|u_{n}\right\|_{\infty} \leqslant \max \left\{\Psi^{-1}(C),-\Psi^{-1}(-C)\right\}
$$

Proof. There are several steps in the proof. First, we will prove that

$$
\begin{aligned}
& \sup _{\tau \in[0, T]} \int_{\Omega}\left[G_{k}\left(\Psi_{n}\left(u_{n}(\tau)\right)\right)\right]^{2}+\int_{Q_{T}}\left|\nabla G_{k}\left(\Psi_{n}\left(u_{n}\right)\right)\right|^{2} \\
& \leqslant C \int_{Q_{T}}|f|\left[G_{k}\left(\left|\Psi_{n}\left(u_{n}\right)\right|\right)\right]^{2}+C k^{2} \int_{\left\{\left|\Psi_{n}\left(u_{n}\right)\right|>k\right\}}|f|,
\end{aligned}
$$

for all $k$ big enough, $C>0$ being a constant that does not depend on $\|f\|_{r, q}$.

To this end, since we can always assume that $u_{0, n}$ is bounded in $L^{\infty}(\Omega)$, we can choose $k$ such that, for every $n, k>\left\|\Psi\left(u_{0, n}\right)\right\|_{L^{\infty}}+\left\|h\left(u_{0, n}\right)\right\|_{L^{\infty}}$, where $h$ is defined as in (28). This implies that $k>\left|\Psi_{n}\left(u_{0, n}\right)\right|$. Then we take $\psi(s)=G_{k}\left(\Psi_{n}(s)\right)$ in (35). Denoting $\phi(s)=\int_{0}^{s} \mathrm{e}^{|\gamma(\sigma)|} G_{k}\left(\Psi_{n}(\sigma)\right) \mathrm{d} \sigma$, one has

$$
\begin{aligned}
& \sup _{\tau \in[0, T]} \int_{\Omega} \phi\left(u_{n}(\tau)\right)+\int_{\left\{\left|\Psi_{n}\left(u_{n}\right)\right|>k\right\}} \mathrm{e}^{\left|\gamma\left(u_{n}\right)\right|} \alpha_{n}\left(u_{n}\right) \Psi_{n}^{\prime}\left(u_{n}\right)\left|\nabla u_{n}\right|^{2} \\
& \quad \leqslant \int_{Q_{T}}|f| \mathrm{e}^{\left|\gamma\left(u_{n}\right)\right|} G_{k}\left(\left|\Psi_{n}\left(u_{n}\right)\right|\right) \leqslant C \int_{Q_{T}}|f|\left(1+\left|\Psi_{n}\left(u_{n}\right)\right|\right) G_{k}\left(\left|\Psi_{n}\left(u_{n}\right)\right|\right),
\end{aligned}
$$


by applying $(\mathrm{C} 1)$.

We prove (51) by analyzing each term in this inequality. Observe first that

$$
\begin{aligned}
\phi(s) & =\int_{0}^{s} \mathrm{e}^{|\gamma(\sigma)|} G_{k}\left(\Psi_{n}(\sigma)\right) \mathrm{d} \sigma \geqslant \frac{1}{\left\|\alpha_{n}\right\|_{\infty}} \int_{0}^{s} \mathrm{e}^{|\gamma(\sigma)|} \alpha_{n}(\sigma) G_{k}\left(\Psi_{n}(\sigma)\right) \mathrm{d} \sigma \\
& =\frac{1}{\left\|\alpha_{n}\right\|_{\infty}} \int_{0}^{s} \Psi_{n}^{\prime}(\sigma) G_{k}\left(\Psi_{n}(\sigma)\right) \mathrm{d} \sigma \geqslant \frac{1}{2\left(\|\alpha\|_{\infty}+1\right)}\left[G_{k}\left(\Psi_{n}(s)\right)\right]^{2} .
\end{aligned}
$$

Moreover, we obviously have

$$
\int_{\left\{\left|\Psi_{n}\left(u_{n}\right)\right|>k\right\}} \mathrm{e}^{\left|\gamma\left(u_{n}\right)\right|} \alpha_{n}\left(u_{n}\right) \Psi_{n}^{\prime}\left(u_{n}\right)\left|\nabla u_{n}\right|^{2}=\int_{\left\{\left|\Psi_{n}\left(u_{n}\right)\right|>k\right\}} \Psi_{n}^{\prime}\left(u_{n}\right)^{2}\left|\nabla u_{n}\right|^{2}=\int_{Q_{T}}\left|\nabla G_{k}\left(\Psi_{n}\left(u_{n}\right)\right)\right|^{2} .
$$

Thus, (52) becomes

$$
\sup _{\tau \in[0, T]} \int_{\Omega}\left[G_{k}\left(\Psi_{n}\left(u_{n}(\tau)\right)\right)\right]^{2}+\int_{Q_{T}}\left|\nabla G_{k}\left(\Psi_{n}\left(u_{n}\right)\right)\right|^{2} \leqslant C \int_{Q_{T}}|f|\left(1+\left|\Psi_{n}\left(u_{n}\right)\right|\right) G_{k}\left(\left|\Psi_{n}\left(u_{n}\right)\right|\right) .
$$

Finally, since Young's inequality implies

$$
\begin{aligned}
G_{k}\left(\left|\Psi_{n}\left(u_{n}\right)\right|\right)+\left|\Psi_{n}\left(u_{n}\right)\right| G_{k}\left(\left|\Psi_{n}\left(u_{n}\right)\right|\right) & =\left[G_{k}\left(\left|\Psi_{n}\left(u_{n}\right)\right|\right)\right]^{2}+(k+1) G_{k}\left(\left|\Psi_{n}\left(u_{n}\right)\right|\right) \\
& \leqslant \frac{3}{2}\left[G_{k}\left(\left|\Psi_{n}\left(u_{n}\right)\right|\right)\right]^{2}+\frac{1}{2}(k+1)^{2} \chi_{\left\{\left|\Psi_{n}\left(u_{n}\right)\right|>k\right\}},
\end{aligned}
$$

it follows from (53) that (51) holds true.

Now, note that our hypothesis $1 / r+N / 2 q<1$ implies $N / q^{\prime}+2 / r^{\prime}>N$ and so there exists $\epsilon>0$ such that $N / q^{\prime}+2 / r^{\prime}=(1+\epsilon) N$. Then, denoting $\rho=2(1+\epsilon) r^{\prime}$ and $\sigma=2(1+\epsilon) q^{\prime}$, we conclude that these parameters satisfy the assumptions of the Gagliardo-Nirenberg lemma.

Our next step is to see that

$$
\left(\int_{0}^{T}\left(\int_{\Omega}\left|G_{k}\left(\Psi_{n}\left(u_{n}\right)\right)\right|^{\sigma}\right)^{\rho / \sigma}\right)^{1 / \rho} \leqslant C k\left(\int_{0}^{T}\left|\left\{x \in \Omega:\left|\Psi_{n}\left(u_{n}(x, t)\right)\right|>k\right\}\right|^{\rho / \sigma} \mathrm{d} t\right)^{(1+\epsilon) / \rho}
$$

holds true. To do this, applying Gagliardo-Nirenberg and Young's inequalities, we deduce from (51) that

$$
\begin{aligned}
\left(\int_{0}^{T}\left(\int_{\Omega}\left|G_{k}\left(\Psi_{n}\left(u_{n}\right)\right)\right|^{\sigma}\right)^{\rho / \sigma}\right)^{2 / \rho} & \leqslant C \sup _{\tau \in[0, T]}\left(\int_{\Omega}\left[G_{k}\left(\Psi_{n}\left(u_{n}(\tau)\right)\right)\right]^{2}\right)^{(\rho-2) / \rho}\left(\int_{Q_{T}}\left|\nabla G_{k}\left(\Psi_{n}\left(u_{n}\right)\right)\right|^{2}\right)^{2 / \rho} \\
& \leqslant C \sup _{\tau \in[0, T]} \int_{\Omega}\left[G_{k}\left(\Psi_{n}\left(u_{n}(\tau)\right)\right)\right]^{2}+C \int_{Q_{T}}\left|\nabla G_{k}\left(\Psi_{n}\left(u_{n}\right)\right)\right|^{2} \\
& \leqslant C \int_{Q_{T}}|f|\left[G_{k}\left(\left|\Psi_{n}\left(u_{n}\right)\right|\right)\right]^{2}+C k^{2} \int_{\left\{\left|\Psi_{n}\left(u_{n}\right)\right|>k\right\}}|f| .
\end{aligned}
$$

As in the proof of Proposition 4.1, we may assume that $\|f\|_{r, q}$ is small. Then the first term in the right-hand side may be absorbed by the left hand one. Indeed, by Hölder's inequality 


$$
\begin{aligned}
C \int_{Q_{T}}|f|\left[G_{k}\left(\left|\Psi_{n}\left(u_{n}\right)\right|\right)\right]^{2} & \leqslant C\|f\|_{r, q}\left\|\left[G_{k}\left(\left|\Psi_{n}\left(u_{n}\right)\right|\right)\right]^{2}\right\|_{r^{\prime}, q^{\prime}} \\
& \leqslant C\|f\|_{r, q}\left(\int_{0}^{T}\left(\int_{\Omega}\left|G_{k}\left(\Psi_{n}\left(u_{n}\right)\right)\right|^{\sigma}\right)^{\rho / \sigma}\right)^{2 / \rho},
\end{aligned}
$$

where this last constant only depends on the previous one, $\epsilon$, meas $\Omega$, and $T$. It follows from (55) that

$$
\left(\int_{0}^{T}\left(\int_{\Omega}\left|G_{k}\left(\Psi_{n}\left(u_{n}\right)\right)\right|^{\sigma}\right)^{\rho / \sigma}\right)^{2 / \rho} \leqslant C k^{2} \int_{\left\{\left|\Psi_{n}\left(u_{n}\right)\right|>k\right\}}|f| .
$$

Note that from now on $C>0$ is a constant that does depend on $\|f\|_{r, q}$.

Now the right-hand side in (56) may be estimated as follows:

$$
\begin{aligned}
\int_{\left\{\left|\Psi_{n}\left(u_{n}\right)\right|>k\right\}}|f| & \leqslant\|f\|_{r, q}\left(\int_{0}^{T}\left|\left\{x \in \Omega:\left|\Psi_{n}\left(u_{n}(x, t)\right)\right|>k\right\}\right|^{r^{\prime} / q^{\prime}} \mathrm{d} t\right)^{1 / r^{\prime}} \\
& \leqslant C\left(\int_{0}^{T}\left|\left\{x \in \Omega:\left|\Psi_{n}\left(u_{n}(x, t)\right)\right|>k\right\}\right|^{\rho / \sigma} \mathrm{d} t\right)^{2(1+\epsilon) / \rho} .
\end{aligned}
$$

Hence, we obtain from (56) that (54) holds. This inequality implies, by ([21], Chapter II, Theorem 6.1), that $\left\|\Psi_{n}\left(u_{n}\right)\right\|_{\infty}<C$, where $C$ only depends on the parameters of problem (6).

\section{Convergence of the approximate solutions}

This section deals with the convergence of the sequence $\left\{u_{n}\right\}_{n}$ of approximate solutions of (34). First of all we will prove that there exists $u$ such that, up to a subsequence, $\left\{u_{n}\right\}_{n}$ converges to $u$, for almost every $(x, t) \in Q_{T}$. Then we will prove the convergence of gradients, namely: we will prove in Proposition 6.2 that the sequence $\left\{\nabla T_{k}\left(u_{n}\right)\right\}_{n}$ strongly converges in $L^{2}\left(Q_{T}\right)$ and, as a consequence, it yields that a subsequence, still denoted by $\left\{\nabla u_{n}\right\}_{n}$, converges to $\nabla u$ for almost all $(x, t) \in Q_{T}$. In Proposition 6.3 we will prove the convergence of the quadratic term in $L^{1}(\Omega)$. Finally, we will see in Proposition 6.4 that $\left\{u_{n}\right\}_{n}$ converges to $u$ in $C\left([0, T] ; L^{1}(\Omega)\right)$, which gives meaning to the initial condition. Once these facts have been proved, it will be easy to finish the proof of Theorem 2.1.

To see the pointwise convergence of the sequence of approximate solutions of a parabolic problem, an Aubin type theorem is usually applied (see [4] and [29]). This can still be done in our framework, provided $\Psi(s)$ has at least linear growth, that is, $\Psi^{\prime}(s) \geqslant c_{0}>0$. However this is not always the case, since $\Psi(s)$ can have a very slow growth (see Remark 2.6). Thus, we have to prove the following compactness result, whose proof is strongly inspired on a result by Alt and Luckhaus [1].

Proposition 6.1. Let $\left\{u_{n}\right\}_{n}$ be a sequence of functions such that

$$
u_{n} \in L^{2}\left(0, T ; H_{0}^{1}(\Omega)\right), \quad\left(u_{n}\right)_{t} \in L^{2}\left(0, T ; H^{-1}(\Omega)\right)
$$

(not necessarily bounded in these spaces). Assume that there exists a continuous and strictly increasing function $\Psi: \mathbb{R} \rightarrow \mathbb{R}$ satisfying

$$
\Psi(0)=0, \quad \lim _{s \rightarrow \pm \infty} \Psi(s)= \pm \infty
$$


such that

$$
\left\{\Psi\left(u_{n}\right)\right\}_{n} \text { is bounded in } L^{2}\left(0, T ; H_{0}^{1}(\Omega)\right) .
$$

Assume moreover that

$$
\left\{\left(u_{n}\right)_{t}\right\}_{n} \text { is bounded in } L^{2}\left(0, T ; H^{-1}(\Omega)\right)+L^{1}\left(Q_{T}\right),
$$

and finally that there exists a continuous function $\Phi: \mathbb{R} \rightarrow[0,+\infty)$ satisfying

$$
\lim _{s \rightarrow \pm \infty} \frac{\Phi(s)}{|s|}=\infty
$$

such that

$$
\left\{\Phi\left(u_{n}\right)\right\}_{n} \text { is bounded in } L^{\infty}\left(0, T ; L^{1}(\Omega)\right) .
$$

Then the sequence $\left\{u_{n}\right\}_{n}$ is relatively compact in $L^{1}\left(Q_{T}\right)$.

Proof. We divide the proof in some steps.

Step 1: Assume that (57) holds. Then it is easy to see that the sequence $\left\{\left(u_{n}(t+h)-u_{n}(t)\right) / h\right\}_{n}$ is bounded in $L^{2}\left(0, T-h ; H^{-1}(\Omega)\right)+L^{1}\left(Q_{T-h}\right)$, uniformly with respect to $n$ and $h$. Therefore there exists a constant $C$ such that

$$
\frac{1}{h} \int_{0}^{T-h} \mathrm{~d} t \int_{\Omega}\left[u_{n}(t+h)-u_{n}(t)\right] T_{1}\left(\Psi\left(u_{n}(t+h)\right)-\Psi\left(u_{n}(t)\right)\right) \leqslant C .
$$

Step 2: For each $M>0$ and $\varepsilon>0$ there exists $\delta=\delta_{\varepsilon, M}$ such that, for every $v, w \in H_{0}^{1}(\Omega)$ satisfying

$$
\begin{aligned}
& \|\Psi(v)\|_{H_{0}^{1}(\Omega)} \leqslant M, \quad\|\Psi(w)\|_{H_{0}^{1}(\Omega)} \leqslant M, \\
& \int_{\Omega} \Phi(v) \leqslant M, \quad \int_{\Omega} \Phi(w) \leqslant M, \\
& \int_{\Omega}(v-w) T_{1}(\Psi(v)-\Psi(w))<\delta,
\end{aligned}
$$

one has

$$
\int_{\Omega}|v-w|<\varepsilon
$$

Indeed, by contradiction, assume that there exist two positive constants $M_{0}$ and $\varepsilon_{0}$ and two sequences $\left\{v_{n}\right\}_{n}$ and $\left\{w_{n}\right\}_{n}$ such that

$$
\begin{aligned}
& \left\|\Psi\left(v_{n}\right)\right\|_{H_{0}^{1}(\Omega)} \leqslant M_{0}, \quad\left\|\Psi\left(w_{n}\right)\right\|_{H_{0}^{1}(\Omega)} \leqslant M_{0}, \\
& \int_{\Omega} \Phi\left(v_{n}\right) \leqslant M_{0}, \quad \int_{\Omega} \Phi\left(w_{n}\right) \leqslant M_{0}, \\
& \int_{\Omega}\left(v_{n}-w_{n}\right) T_{1}\left(\Psi\left(v_{n}\right)-\Psi\left(w_{n}\right)\right) \rightarrow 0, \\
& \int_{\Omega}\left|v_{n}-w_{n}\right| \geqslant \varepsilon_{0} .
\end{aligned}
$$


By Rellich's theorem, the sequences $\left\{\Psi\left(v_{n}\right)\right\}_{n}$ and $\left\{\Psi\left(w_{n}\right)\right\}_{n}$ are relatively compact in $L^{2}(\Omega)$, therefore, up to a subsequence which we shall still denote with the index $n$, one can find $\xi$ and $\eta$ in $L^{2}(\Omega)$ such that

$$
\Psi\left(v_{n}\right) \rightarrow \xi, \quad \Psi\left(w_{n}\right) \rightarrow \eta \quad \text { a.e. in } \Omega .
$$

Therefore, setting $v(x)=\Psi^{-1}(\xi(x))$ and $w(x)=\Psi^{-1}(\eta(x))$, one has

$$
v_{n} \rightarrow v, \quad w_{n} \rightarrow w \quad \text { a.e. in } \Omega .
$$

Applying (58), (60) and De la Vallée Poussin's Theorem, we deduce that

$$
v_{n} \rightarrow v, \quad w_{n} \rightarrow w \quad \text { strongly in } L^{1}(\Omega) .
$$

Using this, we obtain that

$$
\int_{\Omega}\left(v_{n}-w_{n}\right) T_{1}\left(\Psi\left(v_{n}\right)-\Psi\left(w_{n}\right)\right) \rightarrow \int_{\Omega}(v-w) T_{1}(\Psi(v)-\Psi(w)),
$$

therefore, by (61), the last integral is zero. By the strict monotonicity of $\Psi$, this implies that $v=w$ a.e. in $\Omega$, which contradicts (62).

Step 3: We wish to show that

$$
\int_{0}^{T-h} \int_{\Omega}\left|u_{n}(t+h)-u_{n}(t)\right| \stackrel{h \rightarrow 0^{+}}{\longrightarrow} 0 \quad \text { uniformly w.r.t. } n .
$$

For fixed $n, h, M$, we consider the measurable set

$$
\begin{aligned}
E=E_{n, h, M}= & \left\{t \in(0, T-h):\left\|\Psi\left(u_{n}(t+h)\right)\right\|_{H_{0}^{1}(\Omega)}+\left\|\Psi\left(u_{n}(t)\right)\right\|_{H_{0}^{1}(\Omega)}\right. \\
& \left.+\frac{1}{h} \int_{\Omega}\left(u_{n}(t+h)-u_{n}(t)\right) T_{1}\left(\Psi\left(u_{n}(t+h)\right)-\Psi\left(u_{n}(t)\right)\right)>M\right\} .
\end{aligned}
$$

Then in the integral in (63), we can split the parts where $t \in E$ and $t \in E^{c}$. As far as the former is concerned, since $|s| \leqslant \Phi(s)+C$, one has, by the assumption (59),

$$
\int_{E} \mathrm{~d} t \int_{\Omega}\left|u_{n}(t+h)-u_{n}(t)\right| \leqslant \int_{E} \mathrm{~d} t \int_{\Omega}\left[\Phi\left(u_{n}(t+h)\right)+\Phi\left(u_{n}(t)\right)+2 C\right] \leqslant C \mathcal{L}^{1}(E),
$$

where $\mathcal{L}^{1}$ denotes the 1-dimensional Lebesgue measure. Since the quantities which appear in the definition of $E$ have bounded integrals with respect to $t$, one has

$$
\mathcal{L}^{1}(E) \leqslant \frac{C}{M}
$$

Therefore (63) follows from Step 2. Obviously one also has

$$
\int_{h}^{T} \int_{\Omega}\left|u_{n}(t)-u_{n}(t-h)\right| \stackrel{h \rightarrow 0^{+}}{\longrightarrow} \text { uniformly w.r.t. } n \text {. }
$$

Step 4: We wish to approximate $u_{n}$ with functions which are piecewise constants in time. For $M>0$, we define the set

$$
F=F_{M, n}=\left\{t \in(0, T):\left\|\Psi\left(u_{n}(t)\right)\right\|_{H_{0}^{1}(\Omega)}>M\right\}
$$


As before, one has

$$
\mathcal{L}^{1}(F) \leqslant \frac{C}{M} .
$$

Moreover we set

$$
v_{n}(t)=u_{n}(t) \chi_{F^{c}}(t)
$$

We will show that for every $\varepsilon>0$ one can choose $M, h$ (for simplicity of notation we will take a divisor of $T$ ) and $s_{\varepsilon}=s_{\varepsilon, n, M, h} \in(0, h)$ such that

$$
\int_{0}^{T} \int_{\Omega}^{T}\left|u_{n}(t)-\sum_{i=1}^{T / h} v_{n}\left((i-1) h+s_{\varepsilon}\right) \chi_{((i-1) h, i h)}(t)\right|<\varepsilon \quad \text { for every } n .
$$

To do this, we compute the average with respect to $s$ :

$$
\begin{aligned}
& \frac{1}{h} \int_{0}^{h} \mathrm{~d} s \int_{0}^{T} \mathrm{~d} t \int_{\Omega}\left|u_{n}(t)-\sum_{i=1}^{T / h} v_{n}((i-1) h+s) \chi_{((i-1) h, i h)}(t)\right| \\
& \quad=\frac{1}{h} \int_{0}^{h} \mathrm{~d} s \sum_{i=1}^{T / h} \int_{(i-1) h}^{i h} \mathrm{~d} t \int_{\Omega}\left|u_{n}(t)-v_{n}((i-1) h+s)\right| \\
& =\frac{1}{h} \sum_{i=1}^{T / h} \int_{(i-1) h}^{i h} \mathrm{~d} \tau \int_{(i-1) h}^{i h} \mathrm{~d} t \int_{\Omega}\left|u_{n}(t)-v_{n}(\tau)\right| \\
& \leqslant \frac{1}{h} \int_{-h}^{h} \mathrm{~d} \tau \int_{(-\tau) \vee 0}^{(T-\tau) \wedge T} \mathrm{~d} t \int_{\Omega}\left|u_{n}(t)-v_{n}(t+\tau)\right| .
\end{aligned}
$$

We now distinguish between the values $t$ such that $t+\tau \in F$, where $v_{n}(t+\tau)=0$, and those such that $t+\tau \in F^{c}$, where $v_{n}(t+\tau)=u_{n}(t+\tau)$. Therefore

$$
\begin{aligned}
& \frac{1}{h} \int_{0}^{h} \mathrm{~d} s \int_{0}^{T} \mathrm{~d} t \int_{\Omega}\left|u_{n}(t)-\sum_{i=1}^{T / h} v_{n}((i-1) h+s) \chi_{((i-1) h, i h)}(t)\right| \\
& \leqslant \frac{1}{h} \int_{-h}^{h} \mathrm{~d} \tau \int_{(-\tau) \vee 0}^{(T-\tau) \wedge T} \mathrm{~d} t \int_{\Omega}\left|u_{n}(t)-u_{n}(t+\tau)\right|+\frac{1}{h} \int_{-h}^{h} \mathrm{~d} \tau \int_{F} \mathrm{~d} t \int_{\Omega}\left|u_{n}(t)\right| \\
& \quad \leqslant 2 \sup _{|\tau| \leqslant h} \int_{(-\tau) \vee 0}^{(T-\tau) \wedge T} \mathrm{~d} t \int_{\Omega}\left|u_{n}(t)-u_{n}(t+\tau)\right|+2 \int_{F} \mathrm{~d} t \int_{\Omega}\left|u_{n}(t)\right| .
\end{aligned}
$$

If we choose $M$ large enough the latter integral is less than $\varepsilon / 2$, while the previous one can be made smaller than $\varepsilon / 2$ by choosing $h$ small enough, using Step 3 . Thus one can find $s_{\varepsilon}$ such that (64) holds.

Hence, we have shown that for every $\varepsilon$ we can find a sequence $w_{n}^{(\varepsilon)}$ of functions which are constant in time on the intervals $\left((i-1) h_{\varepsilon}, i h_{\varepsilon}\right)$ and such that

$$
\iint_{Q_{T}}\left|u_{n}-w_{n}^{(\varepsilon)}\right|<\varepsilon, \quad\left\|\Psi\left(w_{n}^{(\varepsilon)}\right)\right\|_{L^{\infty}\left(0, T ; H_{0}^{1}(\Omega)\right)} \leqslant M_{\varepsilon} .
$$


Using Rellich's theorem, for every fixed $\varepsilon$ one can extract a subsequence of indices $\left\{m_{n}^{(\varepsilon)}\right\}$ such that $\left\{\Psi\left(w_{m_{n}^{(\varepsilon)}}^{(\varepsilon)}\right)\right\}$ converges strongly in $L^{\infty}\left(0, T ; L^{2}(\Omega)\right)$ and therefore, using the assumption (59), such that $\left\{w_{m_{n}^{(\varepsilon)}}^{(\varepsilon)}\right\}$ converges strongly in $L^{1}\left(Q_{T}\right)$ (in $L^{\infty}\left(0, T ; L^{1}(\Omega)\right.$ ), actually). By repeating the argument for $\varepsilon=1 / k(k \in \mathbb{N})$ and taking a diagonal subsequence, one can find a subsequence of indices $\left\{m_{n}\right\}$ such that, for every $k$,

$$
\begin{aligned}
& \iint_{Q_{T}}\left|u_{m_{n}}-w_{m_{n}}^{(1 / k)}\right|<\frac{1}{k}, \\
& \left\{w_{m_{n}}^{(1 / k)}\right\} \text { converges strongly to some } w^{(1 / k)} \text { in } L^{1}\left(Q_{T}\right) \text { for } n \rightarrow \infty .
\end{aligned}
$$

Step 5: We conclude using Cauchy's criterium: For fixed $\varepsilon>0$ we choose $k>3 / \varepsilon$, then

$$
\left\|u_{m_{n}}-u_{m_{j}}\right\|_{L^{1}\left(Q_{T}\right)} \leqslant\left\|u_{m_{n}}-w_{m_{n}}^{(1 / k)}\right\|_{+}\left\|w_{m_{n}}^{(1 / k)}-w_{m_{j}}^{(1 / k)}\right\|_{+}\left\|w_{m_{j}}^{(1 / k)}-u_{m_{j}}\right\| \leqslant \frac{2 \varepsilon}{3}+\left\|w_{m_{n}}^{(1 / k)}-w_{m_{j}}^{(1 / k)}\right\| .
$$

Now the last norm can be made smaller than $\varepsilon / 3$ by choosing $n$ and $j$ large enough. This concludes the proof of Proposition 6.1.

Corollary 6.1. Assume that (15) and (16) hold true. If $\left\{u_{n}\right\}_{n}$ is a sequence of solutions of the approximate problems (34), then there exist a subsequence, still denoted by $\left\{u_{n}\right\}_{n}$, and a function $u \in L^{1}\left(Q_{T}\right)$ such that

$$
u_{n} \rightarrow u \text { a.e. and strongly in } L^{1}\left(Q_{T}\right) .
$$

Proof. We only have to check that the sequence of approximate solutions satisfies the assumptions of the previous result. Recalling (24) and (29), we have the inequalities $\Psi_{n}^{\prime}(s) \geqslant \Psi^{\prime}(s) \geqslant 0,\left|\Psi_{n}(s)\right| \geqslant|\Psi(s)|$ and $\Phi_{n}(s) \geqslant$ $\Phi(s) \geqslant 0$. Thus,

$$
\left\|\Psi\left(u_{n}\right)\right\|_{L^{2}\left(0, T ; H_{0}^{1}(\Omega)\right)} \leqslant\left\|\Psi_{n}\left(u_{n}\right)\right\|_{L^{2}\left(0, T ; H_{0}^{1}(\Omega)\right)} \leqslant C
$$

by (40), and

$$
\left\|\Phi\left(u_{n}\right)\right\|_{L^{\infty}\left(0, T ; L^{1}(\Omega)\right)} \leqslant\left\|\Phi_{n}\left(u_{n}\right)\right\|_{L^{\infty}\left(0, T ; L^{1}(\Omega)\right)} \leqslant C,
$$

by (38). Furthermore, by Corollary 4.2, the sequence $\left\{\left(u_{n}\right)_{t}\right\}_{n}$ is bounded in $L^{2}\left(0, T ; H^{-1}(\Omega)\right)+L^{1}\left(Q_{T}\right)$.

We will prove now that, for each $k>0$, the sequence $\left\{\nabla T_{k}\left(u_{n}\right)\right\}_{n}$ strongly converges to $\nabla T_{k}(u)$ in $L^{2}\left(Q_{T}\right)$.

Proposition 6.2. Assume that (15) and (16) are satisfied, and let $\left\{u_{n}\right\}_{n}$ be a sequence of solutions of problem (34) which converges to $u$ a.e. and strongly in $L^{1}\left(Q_{T}\right)$. Then, for every fixed $k>0$,

$$
\nabla T_{k}\left(u_{n}\right) \rightarrow \nabla T_{k}(u) \quad \text { strongly in } L^{2}\left(Q_{T}\right) .
$$

Proof. To prove this proposition, we begin by introducing a suitable regularization with respect to time (see [22, 23]). For every $v \in \mathbb{N}$, we define $\left(T_{k}(u)\right)_{v}$ as the solution of the Cauchy problem

$$
\left\{\begin{array}{l}
\frac{1}{v}\left[\left(T_{k}(u)\right)_{v}\right]_{t}+\left(T_{k}(u)\right)_{v}=T_{k}(u) ; \\
\left(T_{k}(u)\right)_{v}(0)=T_{k}\left(u_{0, v}\right) .
\end{array}\right.
$$

Then, using the assumptions (30)-(32) on the approximations of the initial datum, one has (see [22]):

$$
\begin{aligned}
& \left(T_{k}(u)\right)_{v} \in L^{2}\left(0, T ; H_{0}^{1}(\Omega)\right), \quad\left(\left(T_{k}(u)\right)_{\nu}\right)_{t} \in L^{2}\left(0, T ; H_{0}^{1}(\Omega)\right), \\
& \left\|\left(T_{k}(u)\right)_{v}\right\|_{L^{\infty}\left(Q_{T}\right)} \leqslant\left\|T_{k}(u)\right\|_{L^{\infty}\left(Q_{T}\right)} \leqslant k,
\end{aligned}
$$


and as $v$ goes to infinity

$$
\left(T_{k}(u)\right)_{v} \rightarrow T_{k}(u) \quad \text { strongly in } L^{2}\left(0, T ; H_{0}^{1}(\Omega)\right) .
$$

Use the function $v=v_{\nu, n}=\varphi\left(\left(T_{k}\left(u_{n}\right)-\left(T_{k}(u)\right)_{\nu}\right)^{+}\right) \mathrm{e}^{-\gamma\left(T_{k}\left(u_{n}\right)\right)}$ as test in Proposition 3.2(1), where

$$
\varphi(s)=\mathrm{e}^{\lambda s}-1,
$$

and $\lambda>0$ will be conveniently chosen hereafter. Then we obtain

$$
\int_{0}^{T}\left\langle\left(u_{n}\right)_{t}, \mathrm{e}^{\gamma\left(u_{n}\right)} v\right\rangle+\int_{Q_{T}} \mathrm{e}^{\gamma\left(u_{n}\right)} \mathbf{a}_{n}\left(x, t, u_{n}\right) \nabla u_{n} \nabla v \leqslant \int_{Q_{T}} T_{n}(f) \mathrm{e}^{\gamma\left(u_{n}\right)} v .
$$

Now, we estimate the terms of this inequality. For the sake of convenience, we will denote by $\omega(v)$ a quantity which goes to zero as $v$ goes to infinity, while $\omega^{v}(n)$ will denote a quantity which goes to zero as $n$ goes to infinity, for every fixed $v$. On the other hand, to simplify the exposition we divide the proof into various steps.

Step 1: To begin with, one can prove that

$$
\int_{0}^{T}\left\langle\left(u_{n}\right)_{t}, \mathrm{e}^{\gamma\left(u_{n}\right)} v\right\rangle \geqslant \omega^{v}(n)+\omega(v) .
$$

This result may be proved as in Lemma 3 of [13] (see also [23]) with some minor modifications.

Step 2: We will prove that

$$
\int_{Q_{T}} T_{n}(f) \mathrm{e}^{\gamma\left(u_{n}\right)} v \leqslant \omega^{v}(n)
$$

Indeed, using the hypothesis $(\mathbf{C 1})$, we can write

$$
\begin{aligned}
\int_{Q_{T}} T_{n}(f) \mathrm{e}^{\gamma\left(u_{n}\right)} v & =\int_{Q_{T}} T_{n}(f) \varphi\left(\left(T_{k}\left(u_{n}\right)-\left(T_{k}(u)\right)_{\nu}\right)^{+}\right) \mathrm{e}^{\gamma\left(u_{n}\right)-T_{k}\left(\gamma\left(u_{n}\right)\right)} \\
& \leqslant \int_{Q_{T}}|f| \varphi\left(\left(T_{k}\left(u_{n}\right)-\left(T_{k}(u)\right)_{\nu}\right)^{+}\right) \mathrm{e}^{\left|\gamma\left(u_{n}\right)\right|}, \\
& \leqslant C \int_{Q_{T}}|f| \varphi\left(\left(T_{k}\left(u_{n}\right)-\left(T_{k}(u)\right)_{v}\right)^{+}\right)\left(1+\left|\Psi_{n}\left(u_{n}\right)\right|\right) \\
& \leqslant C\left(\frac{3}{2} \int_{Q_{T}}|f| \varphi\left(\left(T_{k}\left(u_{n}\right)-\left(T_{k}(u)\right)_{\nu}\right)^{+}\right)+\frac{1}{2} \int_{Q_{T}}|f| \varphi\left(\left(T_{k}\left(u_{n}\right)-\left(T_{k}(u)\right)_{v}\right)^{+}\right)\left|\Psi_{n}\left(u_{n}\right)\right|^{2}\right) \\
& =\omega^{v}(n)+\omega(v)+C \int_{Q_{T}}|f| \varphi\left(\left(T_{k}\left(u_{n}\right)-\left(T_{k}(u)\right)_{v}\right)^{+}\right)\left|\Psi_{n}\left(u_{n}\right)\right|^{2} \\
& =\omega^{v}(n)+\omega(v)+F .
\end{aligned}
$$

Let us now estimate the integral $F$. Hypothesis (16) on $f$ and Hölder's inequality yield

$$
F \leqslant\left\|f \varphi\left(\left(T_{k}\left(u_{n}\right)-\left(T_{k}(u)\right)_{v}\right)^{+}\right)\right\|_{r, q}\left\|\Psi_{n}\left(u_{n}\right)^{2}\right\|_{r^{\prime}, q^{\prime}} \leqslant\left\|f \varphi\left(\left(T_{k}\left(u_{n}\right)-\left(T_{k}(u)\right)_{v}\right)^{+}\right)\right\|_{r, q}\left\|\Psi_{n}\left(u_{n}\right)\right\|_{2 r^{\prime}, 2 q^{\prime}}^{2} .
$$

Defining $\rho=2 r^{\prime}, \sigma=2 q^{\prime}$, so that $(\rho, \sigma)$ satisfy conditions (36) and (37) of the Gagliardo-Nirenberg Lemma 3.1, the estimates (39) and (40) lead to

$$
F \leqslant C\left\|f \varphi\left(\left(T_{k}\left(u_{n}\right)-\left(T_{k}(u)\right)_{\nu}\right)^{+}\right)\right\|_{r, q}=\omega^{\nu}(n)+\omega(v),
$$


which concludes the proof of Step 2.

Step 3: We will prove that

$$
\int_{\left\{\left|u_{n}\right| \leqslant k\right\}}\left|\nabla\left(T_{k}\left(u_{n}\right)-\left(T_{k}(u)\right)_{\nu}\right)^{+}\right|^{2} \leqslant \omega^{\nu}(n)+\omega(v) .
$$

Thanks to the first two Steps we have proved that

$$
\int_{Q_{T}} \mathrm{e}^{\gamma\left(u_{n}\right)} \mathbf{a}_{n}\left(x, t, u_{n}\right) \nabla u_{n} \nabla v \leqslant \omega^{\nu}(n)+\omega(v) .
$$

Let us now estimate the left-hand side. From now on we will write $\mathbf{a}_{n}\left(u_{n}\right)$ instead of $\mathbf{a}_{n}\left(x, t, u_{n}\right)$. We can write

$$
\begin{aligned}
\int_{Q_{T}} & \mathrm{e}^{\gamma\left(u_{n}\right)} \mathbf{a}_{n}\left(u_{n}\right) \nabla u_{n} \nabla v \\
= & \int_{Q_{T}} \mathbf{a}_{n}\left(u_{n}\right) \nabla u_{n} \nabla\left(\left(T_{k}\left(u_{n}\right)-\left(T_{k}(u)\right)_{\nu}\right)^{+}\right) \varphi^{\prime}\left(\left(T_{k}\left(u_{n}\right)-\left(T_{k}(u)\right)_{\nu}\right)^{+}\right) \mathrm{e}^{\gamma\left(u_{n}\right)-\gamma\left(T_{k}\left(u_{n}\right)\right)} \\
& -\int_{\left\{\left|u_{n}\right| \leqslant k\right\}} \mathbf{a}_{n}\left(u_{n}\right) \nabla u_{n} \nabla u_{n} \frac{\beta\left(u_{n}\right)}{\alpha\left(u_{n}\right)} \varphi\left(\left(T_{k}\left(u_{n}\right)-\left(T_{k}(u)\right)_{\nu}\right)^{+}\right) \\
= & \int_{\left\{\left|u_{n}\right| \leqslant k\right\}} \mathbf{a}_{n}\left(u_{n}\right) \nabla\left(T_{k}\left(u_{n}\right)-\left(T_{k}(u)\right)_{\nu}\right) \nabla\left(T_{k}\left(u_{n}\right)-T_{k}(u)_{\nu}\right)^{+} \varphi^{\prime}\left(\left(T_{k}\left(u_{n}\right)-\left(T_{k}(u)\right)_{\nu}\right)^{+}\right) \\
& +\int_{\left\{\left|u_{n}\right| \leqslant k\right\}} \mathbf{a}_{n}\left(u_{n}\right) \nabla\left(T_{k}(u)\right)_{\nu} \nabla\left(T_{k}\left(u_{n}\right)-\left(T_{k}(u)\right)_{\nu}\right)^{+} \varphi^{\prime}\left(\left(T_{k}\left(u_{n}\right)-\left(T_{k}(u)\right)_{\nu}\right)^{+}\right) \\
& -\int_{\left\{u_{n}>k\right\}} \mathbf{a}_{n}\left(u_{n}\right) \nabla u_{n} \nabla\left(T_{k}(u)\right)_{\nu} \varphi^{\prime}\left(\left(T_{k}\left(u_{n}\right)-\left(T_{k}(u)\right)_{\nu}\right)^{+}\right) \mathrm{e}^{\gamma\left(u_{n}\right)-\gamma\left(T_{k}\left(u_{n}\right)\right)} \\
& -\int_{\left\{\left|u_{n}\right| \leqslant k\right\}} \mathbf{a}_{n}\left(u_{n}\right) \nabla u_{n} \nabla u_{n} \frac{\beta\left(u_{n}\right)}{\alpha\left(u_{n}\right)} \varphi\left(\left(T_{k}\left(u_{n}\right)-\left(T_{k}(u)\right)_{\nu}\right)^{+}\right) \\
= & A_{1}+A_{2}+A_{3}+A_{4} .
\end{aligned}
$$

Now,

$$
\begin{aligned}
A_{2}= & \int_{\{|u| \neq k\}} \mathbf{a}_{n}\left(u_{n}\right) \nabla\left(T_{k}(u)\right)_{\nu} \nabla\left(T_{k}\left(u_{n}\right)-\left(T_{k}(u)\right)_{\nu}\right)^{+} \varphi^{\prime}\left(\left(T_{k}\left(u_{n}\right)-\left(T_{k}(u)\right)_{\nu}\right)^{+}\right) \chi_{\left\{\left|u_{n}\right| \leqslant k\right\}} \\
& +\int_{\{|u|=k\}} \mathbf{a}_{n}\left(u_{n}\right) \nabla\left(T_{k}(u)\right)_{\nu} \nabla\left(T_{k}\left(u_{n}\right)-\left(T_{k}(u)\right)_{\nu}\right)^{+} \varphi^{\prime}\left(\left(T_{k}\left(u_{n}\right)-\left(T_{k}(u)\right)_{\nu}\right)^{+}\right) \chi_{\left\{\left|u_{n}\right| \leqslant k\right\}} \\
= & \omega(v)+\omega^{\nu}(n) \\
& +\int_{\{|u|=k\}} \mathbf{a}_{n}\left(u_{n}\right) \nabla\left(T_{k}(u)\right)_{\nu} \nabla\left(\left(T_{k}\left(u_{n}\right)-\left(T_{k}(u)\right)_{\nu}\right)^{+}\right) \varphi^{\prime}\left(\left(T_{k}\left(u_{n}\right)-\left(T_{k}(u)\right)_{\nu}\right)^{+}\right) \chi_{\left\{\left|u_{n}\right| \leqslant k\right\}} .
\end{aligned}
$$

Here we have used the weak convergence of $\nabla T_{k}\left(u_{n}\right)$ to $\nabla T_{k}(u)$ in $L^{2}\left(Q_{T} ; \mathbb{R}^{N}\right)$, the strong convergence of $\nabla\left(T_{k}(u)\right)_{v}$ to $\nabla T_{k}(u)$ in $L^{2}\left(Q_{T} ; \mathbb{R}^{N}\right)$ and the fact that $\chi_{\left\{\left|u_{n}\right| \leqslant k\right\}} \chi_{\{|u| \neq k\}}$ converges to $\chi_{\{|u|<k\}}$ almost everywhere. Moreover, by Hölder's inequality, we have 


$$
\begin{aligned}
& \left|\int_{\{|u|=k\}} \mathbf{a}_{n}\left(u_{n}\right) \nabla\left(T_{k}(u)\right)_{v} \nabla\left(T_{k}\left(u_{n}\right)-\left(T_{k}(u)\right)_{\nu}\right)^{+} \varphi^{\prime}\left(\left(T_{k}\left(u_{n}\right)-\left(T_{k}(u)\right)_{v}\right)^{+}\right) \chi_{\left\{\left|u_{n}\right| \leqslant k\right\}}\right| \\
& \quad \leqslant C(k)\left(\int_{Q_{T}}\left|\nabla\left(\left(T_{k}\left(u_{n}\right)-\left(T_{k}(u)\right)_{\nu}\right)^{+}\right)\right|^{2}\right)^{1 / 2}\left(\int_{\{|u|=k\}}\left|\nabla\left(T_{k}(u)\right)_{\nu}\right|^{2}\right)^{1 / 2} \leqslant C(k) \omega(v) .
\end{aligned}
$$

In conclusion

$$
\left|A_{2}\right| \leqslant \omega^{v}(n)+\omega(v) .
$$

Applying (9) and (40) we can estimate $A_{3}$ in the following way:

$$
\begin{aligned}
\left|A_{3}\right| & \leqslant \int_{\left\{u_{n}>k\right\}}\left|\mathbf{a}_{n}\left(u_{n}\right) \nabla u_{n} \nabla\left(T_{k}(u)\right)_{\nu} \varphi^{\prime}\left(\left(T_{k}\left(u_{n}\right)-\left(T_{k}(u)\right)_{\nu}\right)^{+}\right) \mathrm{e}^{\gamma\left(u_{n}\right)-\gamma\left(T_{k}\left(u_{n}\right)\right)}\right| \\
& \leqslant C(k) \int_{\left\{u_{n}>k\right\}}\left|\nabla \Psi_{n}\left(u_{n}\right)\right|\left|\nabla\left(T_{k}(u)\right)_{v}\right| \leqslant C(k)\left(\int_{\left\{u_{n}>k\right\}}\left|\nabla\left(T_{k}(u)\right)_{v}\right|^{2}\right)^{1 / 2} \\
& \leqslant C(k)\left(\int_{\{u \neq k\}}\left|\nabla\left(T_{k}(u)\right)_{v}\right|^{2} \chi_{\left\{u_{n}>k\right\}}+\int_{\{u=k\}}\left|\nabla\left(T_{k}(u)\right)_{v}\right|^{2} \chi_{\left\{u_{n}>k\right\}}\right)^{1 / 2} \\
& \leqslant C(k)\left(\int_{\{u>k\}}\left|\nabla\left(T_{k}(u)\right)_{v}\right|^{2}+\omega(n)+\int_{\{u=k\}}\left|\nabla\left(T_{k}(u)\right)_{v}\right|^{2}\right)^{1 / 2} \\
& =\omega^{v}(n)+\omega(v),
\end{aligned}
$$

by Lebesgue's Theorem. As far as the term $A_{1}$ is concerned, using the first inequality in (25), we obtain

$$
\begin{aligned}
A_{1} & \geqslant \int_{\left\{\left|u_{n}\right| \leqslant k\right\}} \alpha_{n}\left(u_{n}\right)\left|\nabla\left(T_{k}\left(u_{n}\right)-\left(T_{k}(u)\right)_{\nu}\right)^{+}\right|^{2} \varphi^{\prime}\left(\left(T_{k}\left(u_{n}\right)-\left(T_{k}(u)\right)_{\nu}\right)^{+}\right) \\
& \geqslant C_{1}(k) \int_{\left\{\left|u_{n}\right| \leqslant k\right\}}\left|\nabla\left(T_{k}\left(u_{n}\right)-\left(T_{k}(u)\right)_{\nu}\right)^{+}\right|^{2} \varphi^{\prime}\left(\left(T_{k}\left(u_{n}\right)-\left(T_{k}(u)\right)_{\nu}\right)^{+}\right),
\end{aligned}
$$

for some positive $C_{1}(k)$. We only have to deal with the term $A_{4}$ :

$$
\begin{aligned}
A_{4}= & -\int_{\left\{\left|u_{n}\right| \leqslant k\right\}} \mathbf{a}_{n}\left(u_{n}\right) \nabla\left(T_{k}\left(u_{n}\right)-\left(T_{k}(u)\right)_{v}\right)^{+} \nabla\left(T_{k}\left(u_{n}\right)-\left(T_{k}(u)\right)_{\nu}\right)^{+} \frac{\beta\left(u_{n}\right)}{\alpha\left(u_{n}\right)} \varphi\left(\left(T_{k}\left(u_{n}\right)-\left(T_{k}(u)\right)_{\nu}\right)^{+}\right) \\
& -\int_{\left\{\left|u_{n}\right| \leqslant k\right\}} \mathbf{a}_{n}\left(u_{n}\right) \nabla\left(T_{k}(u)\right)_{\nu} \nabla\left(T_{k}\left(u_{n}\right)-\left(T_{k}(u)\right)_{\nu}\right)^{+} \frac{\beta\left(u_{n}\right)}{\alpha\left(u_{n}\right)} \varphi\left(\left(T_{k}\left(u_{n}\right)-\left(T_{k}(u)\right)_{v}\right)^{+}\right) \\
& -\int_{\left\{\left|u_{n}\right| \leqslant k\right\}} \mathbf{a}_{n}\left(u_{n}\right) \nabla T_{k}\left(u_{n}\right) \nabla\left(T_{k}(u)\right)_{\nu} \frac{\beta\left(u_{n}\right)}{\alpha\left(u_{n}\right)} \varphi\left(\left(T_{k}\left(u_{n}\right)-\left(T_{k}(u)\right)_{\nu}\right)^{+} .\right.
\end{aligned}
$$

Using techniques similar to the ones employed above, the last two integrals can be easily shown to be equal to $\omega^{v}(n)+\omega(v)$. Therefore we can write

$$
A_{4} \geqslant-C_{2}(k) \int_{\left\{\left|u_{n}\right| \leqslant k\right\}}\left|\nabla\left(T_{k}\left(u_{n}\right)-\left(T_{k}(u)\right)_{\nu}\right)^{+}\right|^{2}\left|\varphi\left(\left(T_{k}\left(u_{n}\right)-\left(T_{k}(u)\right)_{\nu}\right)^{+}\right)\right|+\omega^{\nu}(n)+\omega(v) .
$$


This is where we use the function $\varphi$. Indeed we can choose the parameter $\lambda=\lambda(k)$ in the definition of $\varphi$ such that

$$
C_{2}(k)|\varphi(s)| \leqslant \frac{C_{1}(k)}{2} \varphi^{\prime}(s)
$$

for every $s \in \mathbb{R}$. Therefore

$$
A_{4} \geqslant-\frac{1}{2} A_{1}+\omega^{v}(n)+\omega(v) .
$$

Putting together formulas (66)-(70), we get (65).

Step 4: Using $v=-\varphi\left(\left(T_{k}\left(u_{n}\right)-\left(T_{k}(u)\right)_{\nu}\right)^{-}\right) \mathrm{e}^{-\gamma\left(T_{k}\left(u_{n}\right)\right)}$ as test function in Proposition 3.2(2), and working exactly as in the previous step, we obtain the analogous of (65),

$$
\int_{\left\{\left|u_{n}\right| \leqslant k\right\}}\left|\nabla\left(T_{k}\left(u_{n}\right)-\left(T_{k}(u)\right)_{v}\right)^{-}\right|^{2} \leqslant \omega^{\nu}(n)+\omega(v) .
$$

The two estimates (65) and (71) lead to

$$
\int_{\left\{\left|u_{n}\right| \leqslant k\right\}}\left|\nabla\left(T_{k}\left(u_{n}\right)-\left(T_{k}(u)\right)_{\nu}\right)\right|^{2} \leqslant \omega^{\nu}(n)+\omega(v) .
$$

Step 5: Adding and subtracting $\nabla T_{k}(u)$ from (72), we deduce that, for every $k>0$,

$$
\int_{\left\{\left|u_{n}\right| \leqslant k\right\}}\left|\nabla\left(T_{k}\left(u_{n}\right)-T_{k}(u)\right)\right|^{2} \leqslant \omega^{v}(n)+\omega(v),
$$

and, finally,

$$
\int_{Q_{T}}\left|\nabla\left(T_{k}\left(u_{n}\right)-T_{k}(u)\right)\right|^{2} \leqslant \omega^{v}(n)+\omega(v)+\int_{\left\{\left|u_{n}\right|>k\right\}}\left|\nabla T_{k}(u)\right|^{2}=\omega^{v}(n)+\omega(v)+\omega(n),
$$

which concludes the proof.

The following result follows easily from Corollary 6.1, Proposition 6.2, Corollary 4.1:

Corollary 6.2. One can extract a subsequence, still denoted by $\left\{u_{n}\right\}_{n}$, such that

$$
\begin{aligned}
& u_{n} \rightarrow u \quad \text { a.e. in } Q_{T} \text { and strongly in } L^{1}\left(Q_{T}\right), \\
& \nabla u_{n} \rightarrow \nabla u \quad \text { a.e. in } Q_{T}, \\
& \nabla T_{k}\left(u_{n}\right) \rightarrow \nabla T_{k}(u) \quad \text { strongly in } L^{2}\left(Q_{T} ; \mathbb{R}^{N}\right), \text { for every } k>0, \\
& \mathbf{a}_{n}\left(u_{n}\right) \nabla u_{n} \rightarrow \mathbf{a}(u) \nabla u \quad \text { weakly in } L^{2}\left(Q_{T} ; \mathbb{R}^{N}\right), \\
& \mathbf{a}_{n}\left(u_{n}\right) \nabla u_{n} \rightarrow \mathbf{a}(u) \nabla u \quad \text { strongly in } L^{q}\left(Q_{T} ; \mathbb{R}^{N}\right), \text { for every } 1 \leqslant q<2, \\
& T_{n}\left(b\left(u_{n}, \nabla u_{n}\right)\right) \rightarrow b(u, \nabla u) \quad \text { a.e. in } Q_{T} .
\end{aligned}
$$

From now on we will assume that $\left\{u_{n}\right\}$ is a subsequence like in the previous statement.

Proposition 6.3. Assuming that (15) and (16) hold true, then

$$
T_{n}\left(b\left(x, t, u_{n}, \nabla u_{n}\right)\right) \rightarrow b(x, t, u, \nabla u) \quad \text { in } L^{1}\left(Q_{T}\right) .
$$


Proof. By (75), using Vitali's theorem, we only have to prove the equi-integrability of the sequence $\left\{T_{n}\left(b\left(u_{n}\right.\right.\right.$, $\left.\left.\left.\nabla u_{n}\right)\right)\right\}_{n}$.

Let $E$ be a measurable subset of $Q_{T}$; then

$$
\begin{aligned}
\int_{E}\left|T_{n}\left(b\left(u_{n}, \nabla u_{n}\right)\right)\right| & \leqslant \int_{E \cap\left\{\left|u_{n}\right| \leqslant k\right\}}\left|T_{n}\left(b\left(u_{n}, \nabla u_{n}\right)\right)\right|+\int_{E \cap\left\{\left|u_{n}\right|>k\right\}}\left|T_{n}\left(b\left(u_{n}, \nabla u_{n}\right)\right)\right| \\
& \leqslant \int_{E} \beta\left(u_{n}\right)\left|\nabla T_{k}\left(u_{n}\right)\right|^{2}+\int_{\left\{\left|u_{n}\right|>k\right\}}\left|T_{n}\left(b\left(u_{n}, \nabla u_{n}\right)\right)\right| \\
& \leqslant\left(\max _{|s| \leqslant k} \beta(s)\right) \int_{E}\left|\nabla T_{k}\left(u_{n}\right)\right|^{2}+C\left\|f \chi_{\left\{\left|u_{n}\right|>k\right\}}\right\|_{r, q}+C \int_{\Omega \cap\left\{\left|u_{0, n}\right|>k\right\}} \Phi\left(u_{0, n}\right),
\end{aligned}
$$

for $k$ large enough by Proposition 4.2. It follows from $f \in L^{r}\left(0, T ; L^{q}(\Omega)\right)$ and $\Phi\left(u_{0, n}\right) \rightarrow \Phi\left(u_{0}\right)$ in $L^{1}(\Omega)$ that

$$
\lim _{k \rightarrow \infty}\left[\left\|f \chi_{\left\{\left|u_{n}\right| \geqslant k\right\}}\right\|_{r, q}+\int_{\Omega \cap\left\{\left|u_{0, n}\right|>k\right\}} \Phi\left(u_{0, n}\right)\right]=0
$$

uniformly with respect to $n$. Thus, for every $\varepsilon>0$, we may fix $k$ satisfying

$$
\int_{E}\left|T_{n}\left(b\left(u_{n}, \nabla u_{n}\right)\right)\right| \leqslant\left(\max _{|s| \leqslant k} \beta(s)\right) \int_{E}\left|\nabla T_{k}\left(u_{n}\right)\right|^{2}+\frac{\varepsilon}{2} .
$$

Therefore, the equi-integrability of the sequence $\left\{\left|\nabla T_{k}\left(u_{n}\right)\right|^{2}\right\}_{n}$ implies that of $\left\{T_{n}\left(b\left(u_{n}, \nabla u_{n}\right)\right)\right\}$, and so Proposition 6.3 is proved.

Proposition 6.4. We assume that (15) and (16) hold true. Then

$$
u_{n} \rightarrow u \quad \text { strongly in } C\left([0, T] ; L^{1}(\Omega)\right) .
$$

Proof. Recalling that $u_{n} \in C\left([0, T] ; L^{1}(\Omega)\right)$, we just have to see that

$$
u_{n} \rightarrow u \quad \text { strongly in } L^{\infty}\left(0, T ; L^{1}(\Omega)\right) .
$$

We begin by denoting $J_{1}(s)=\int_{0}^{s} T_{1}(\sigma) \mathrm{d} \sigma$ and pointing out that

$$
\frac{1}{2}\left(s^{2} \chi_{\{|s|<1\}}+|s| \chi_{\{|s| \geqslant 1\}}\right) \leqslant J_{1}(s) \leqslant|s| \quad \text { for all } s \in \mathbb{R} .
$$

Let us fix $t \in[0, T]$ and take $T_{1}\left(u_{n}-\left(T_{k}(u)\right)_{v}\right) \chi_{(0, t)}$ as test function in the weak formulation of (34), where $\left(T_{k}(u)\right)_{v}$ is the regularization with respect to time of $T_{k}(u)$ introduced in the proof of Proposition 6.2. Then

$$
\begin{gathered}
\int_{0}^{t}\left\langle\left(u_{n}\right)_{t}, T_{1}\left(u_{n}-\left(T_{k}(u)\right)_{\nu}\right)\right\rangle \mathrm{d} \tau+\int_{Q_{t}} \mathbf{a}_{n}\left(u_{n}\right) \nabla u_{n} \nabla T_{1}\left(u_{n}-\left(T_{k}(u)\right)_{\nu}\right) \\
=\int_{Q_{t}}\left(T_{n}\left(b\left(u_{n}, \nabla u_{n}\right)\right)+T_{n}(f)\right) T_{1}\left(u_{n}-\left(T_{k}(u)\right)_{\nu}\right) .
\end{gathered}
$$

Hence, adding and subtracting $\int_{Q_{t}}\left(\left(T_{k}(u)\right)_{v}\right)_{t} T_{1}\left(u_{n}-\left(T_{k}(u)\right)_{v}\right)$ and integrating by parts, we obtain 


$$
\begin{gathered}
\int_{\Omega} J_{1}\left(u_{n}(t)-\left(T_{k}(u)\right)_{v}(t)\right)+\int_{Q_{t}}\left(\left(T_{k}(u)\right)_{\nu}\right)_{t} T_{1}\left(u_{n}-\left(T_{k}(u)\right)_{\nu}\right)+\int_{Q_{t}} \mathbf{a}_{n}\left(u_{n}\right) \nabla u_{n} \nabla T_{1}\left(u_{n}-\left(T_{k}(u)\right)_{\nu}\right) \\
=\int_{Q_{t}}\left(T_{n}\left(b\left(u_{n}, \nabla u_{n}\right)\right)+T_{n}(f)\right) T_{1}\left(u_{n}-\left(T_{k}(u)\right)_{v}\right)+\int_{\Omega} J_{1}\left(u_{0, n}-T_{k}\left(u_{0, v}\right)\right) .
\end{gathered}
$$

Our aim is to estimate $\sup _{t \in[0, T]} \int_{\Omega} J_{1}\left(u_{n}(t)-\left(T_{k}(u)\right)_{v}(t)\right)$ and, to this end, we begin by analyzing the left-hand side of (78). First observe that

$$
\begin{aligned}
& \int_{Q_{t}}\left(\left(T_{k}(u)\right)_{\nu}\right)_{t} T_{1}\left(u_{n}-\left(T_{k}(u)\right)_{\nu}\right) \\
& \quad=\int_{Q_{t}}\left(\left(T_{k}(u)\right)_{\nu}\right)_{t} T_{1}\left(u-\left(T_{k}(u)\right)_{v}\right)+\int_{Q_{t}}\left(\left(T_{k}(u)\right)_{\nu}\right)_{t}\left(T_{1}\left(u_{n}-\left(T_{k}(u)\right)_{\nu}\right)-T_{1}\left(u-\left(T_{k}(u)\right)_{v}\right)\right) \\
& =\int_{Q_{t}}\left(\left(T_{k}(u)\right)_{\nu}\right)_{t} T_{1}\left(u-\left(T_{k}(u)\right)_{v}\right)+\omega^{k, v}(n) .
\end{aligned}
$$

Since $\left|\left(T_{k}(u)\right)_{v}\right| \leqslant k$, the functions $T_{k}(u)-\left(T_{k}(u)\right)_{v}$ and $u-\left(T_{k}(u)\right)_{v}$ have the same sign, so that, by the definition of $\left(T_{k}(u)\right)_{\nu}$,

$$
\left(\left(T_{k}(u)\right)_{\nu}\right)_{t} T_{1}\left(u-\left(T_{k}(u)\right)_{\nu}\right)=v\left(T_{k}(u)-\left(T_{k}(u)\right)_{\nu}\right) T_{1}\left(u-\left(T_{k}(u)\right)_{v}\right) \geqslant 0 .
$$

Thus,

$$
\int_{Q_{t}}\left(\left(T_{k}(u)\right)_{v}\right)_{t} T_{1}\left(u_{n}-\left(T_{k}(u)\right)_{v}\right) \geqslant \omega^{k, v}(n) .
$$

On the other hand, performing easy computations, we have

$$
\begin{aligned}
\int_{Q_{t}} & \mathbf{a}_{n}\left(u_{n}\right) \nabla u_{n} \nabla T_{1}\left(u_{n}-\left(T_{k}(u)\right)_{v}\right) \\
& =\int_{Q_{t} \cap\left\{\left|u_{n}-\left(T_{k}(u)\right)_{v}\right|<1\right\}} \mathbf{a}_{n}\left(u_{n}\right) \nabla u_{n} \nabla\left(u_{n}-T_{k}\left(u_{n}\right)\right)+\int_{Q_{t} \cap\left\{\left|u_{n}-\left(T_{k}(u)\right)_{v}\right|<1\right\}} \mathbf{a}_{n}\left(u_{n}\right) \nabla u_{n} \nabla\left(T_{k}\left(u_{n}\right)-\left(T_{k}(u)\right)_{v}\right) \\
& \geqslant \int_{Q_{t} \cap\left\{\left|u_{n}-\left(T_{k}(u)\right)_{v}\right|<1\right\}} \mathbf{a}_{n}\left(u_{n}\right) \nabla u_{n} \nabla\left(T_{k}\left(u_{n}\right)-\left(T_{k}(u)\right)_{v}\right) .
\end{aligned}
$$

Having in mind (79) and (80), Eq. (78) becomes

$$
\begin{gathered}
\int_{\Omega} J_{1}\left(u_{n}(t)-\left(T_{k}(u)\right)_{v}(t)\right)+\omega^{k, v}(n)+\int_{Q_{t} \cap\left\{\left|u_{n}-\left(T_{k}(u)\right)_{v}\right|<1\right\}} \mathbf{a}_{n}\left(u_{n}\right) \nabla u_{n} \nabla\left(T_{k}\left(u_{n}\right)-\left(T_{k}(u)\right)_{v}\right) \\
\leqslant \int_{Q_{t}}\left(T_{n}\left(b\left(u_{n}, \nabla u_{n}\right)\right)+T_{n}(f)\right) T_{1}\left(u_{n}-\left(T_{k}(u)\right)_{v}\right)+\int_{\Omega} J_{1}\left(u_{0, n}-T_{k}\left(u_{0, v}\right)\right),
\end{gathered}
$$

so that 


$$
\begin{aligned}
& \int_{\Omega} J_{1}\left(u_{n}(t)-\left(T_{k}(u)\right)_{v}(t)\right) \leqslant \int_{Q_{T}}\left|\mathbf{a}_{n}\left(u_{n}\right) \nabla u_{n}\right|\left|\nabla\left(T_{k}\left(u_{n}\right)-\left(T_{k}(u)\right)_{v}\right)\right| \\
& \quad+\int_{Q_{T}}\left|T_{n}\left(b\left(u_{n}, \nabla u_{n}\right)\right)+T_{n}(f)\right|\left|T_{1}\left(u_{n}-\left(T_{k}(u)\right)_{v}\right)\right|+\int_{\Omega}\left|u_{0, n}-T_{k}\left(u_{0, v}\right)\right|+\omega^{k, v}(n) .
\end{aligned}
$$

Therefore, since this inequality holds uniformly on $t \in[0, T]$, we obtain

$$
\sup _{t \in[0, T]} \int_{\Omega} J_{1}\left(u_{n}(t)-\left(T_{k}(u)\right)_{v}(t)\right) \leqslant I_{1}+I_{2}+I_{3}+\omega^{k, v}(n) .
$$

Now, we are going to estimate each term in the above equality. To handle $I_{1}$, recall that

$$
\mathbf{a}_{n}\left(u_{n}\right) \nabla u_{n} \rightarrow \mathbf{a}(u) \nabla u \quad \text { weakly in } L^{2}\left(Q_{T}\right) \text { and a.e. in } Q_{T},
$$

which implies

$$
\left|\mathbf{a}_{n}\left(u_{n}\right) \nabla u_{n}\right| \rightarrow|\mathbf{a}(u) \nabla u| \quad \text { weakly in } L^{2}\left(Q_{T}\right) .
$$

This fact and the strong convergence of truncations yield

$$
I_{1}=\int_{Q_{T}}|\mathbf{a}(u) \nabla u|\left|\nabla\left(T_{k}(u)-\left(T_{k}(u)\right)_{v}\right)\right|+\omega^{k, v}(n)=\omega^{k}(v)+\omega^{k, v}(n) .
$$

The estimate on the second term $I_{2}$ is an easy consequence of the strong convergence in $L^{1}\left(Q_{T}\right)$ of the sequence $\left\{T_{n}\left(b\left(u_{n}, \nabla u_{n}\right)\right)+T_{n}(f)\right\}_{n}$ (see Proposition 6.3):

$$
\begin{aligned}
I_{2} & =\int_{Q_{T}}|b(u, \nabla u)+f|\left|T_{1}\left(u-\left(T_{k}(u)\right)_{v}\right)\right|+\omega^{k, v}(n) \\
& =\int_{Q_{T}}|b(u, \nabla u)+f|\left|T_{1}\left(u-T_{k}(u)\right)\right|+\omega^{k, v}(n)+\omega^{k}(v) \\
& =\omega^{k, v}(n)+\omega^{k}(v)+\omega(k) .
\end{aligned}
$$

Finally, it is straightforward that

$$
I_{3}=\int_{\Omega}\left|u_{0, n}-T_{k}\left(u_{0, v}\right)\right|=\omega^{k, v}(n)+\omega^{k}(v)+\omega(k) .
$$

Thus, it follows from (81) that

$$
\sup _{t \in[0, T]} \int_{\Omega} J_{1}\left(u_{n}(t)-\left(T_{k}(u)\right)_{\nu}(t)\right) \leqslant \omega^{k, v}(n)+\omega^{k}(v)+\omega(k) .
$$

Thanks to (77), we deduce that

$$
\begin{aligned}
& \sup _{t \in[0, T]]_{\left\{\left|u_{n}(t)-\left(T_{k}(u)\right)_{v}(t)\right|<1\right\}}}\left|u_{n}(t)-\left(T_{k}(u)\right)_{v}(t)\right|^{2} \\
& \quad+\sup _{t \in[0, T]_{\left\{\left|u_{n}(t)-\left(T_{k}(u)\right)_{v}(t)\right| \geqslant 1\right\}}}\left|u_{n}(t)-\left(T_{k}(u)\right)_{v}(t)\right| \leqslant \omega^{k, v}(n)+\omega^{k}(v)+\omega(k) .
\end{aligned}
$$

From here, applying Hölder's inequality, a uniform $L^{1}$ estimate follows: 


$$
\begin{aligned}
& \sup _{t \in[0, T]} \int_{\Omega}\left|u_{n}(t)-\left(T_{k}(u)\right)_{v}(t)\right| \\
& \leqslant \sup _{t \in[0, T]_{\left\{\left|u_{n}(t)-\left(T_{k}(u)\right)_{v}(t)\right|<1\right\}}}\left|u_{n}(t)-\left(T_{k}(u)\right)_{v}(t)\right|+\sup _{t \in[0, T]_{\left\{\left|u_{n}(t)-\left(T_{k}(u)\right)_{v}(t)\right| \geqslant 1\right\}}}\left|u_{n}(t)-\left(T_{k}(u)\right)_{v}(t)\right| \\
& \leqslant \sqrt{\operatorname{meas}(\Omega)} \sup _{t \in[0, T]}\left(\int_{\left\{\left|u_{n}(t)-\left(T_{k}(u)\right)_{v}(t)\right|<1\right\}}\left|u_{n}(t)-\left(T_{k}(u)\right)_{v}(t)\right|^{2}\right)^{1 / 2} \\
& +\sup _{t \in[0, T]} \int_{\left\{\left|u_{n}(t)-\left(T_{k}(u)\right)_{v}(t)\right| \geqslant 1\right\}}\left|u_{n}(t)-\left(T_{k}(u)\right)_{v}(t)\right| \\
& \leqslant \omega^{k, v}(n)+\omega^{k}(v)+\omega(k) .
\end{aligned}
$$

Therefore, given $\varepsilon>0$, we may find $k$ and $v$ such that $\omega^{k}(v)+\omega(k)<\varepsilon$. Now, we can choose $n_{0} \in \mathbb{N}$ such that $n \geqslant n_{0}$ implies $\omega^{k, v}(n)<\varepsilon$ and consequently

$$
\sup _{t \in[0, T]} \int_{\Omega}\left|u_{n}(t)-\left(T_{k}(u)\right)_{v}(t)\right| \leqslant 2 \varepsilon .
$$

Thus, if $m, n \geqslant n_{0}$, one has

$$
\sup _{t \in[0, T]} \int_{\Omega}\left|u_{n}(t)-u_{m}(t)\right| \leqslant \sup _{t \in[0, T]} \int\left|u_{\Omega}(t)-\left(T_{k}(u)\right)_{v}(t)\right|+\sup _{t \in[0, T]} \int_{\Omega}\left|u_{m}(t)-\left(T_{k}(u)\right)_{v}(t)\right| \leqslant 4 \varepsilon .
$$

Therefore, $\left\{u_{n}\right\}_{n}$ is a Cauchy sequence in $L^{\infty}\left(0, T ; L^{1}(\Omega)\right)$ and consequently Proposition 6.4 holds true.

We can now prove Theorem 2.1.

Proof of Theorem 2.1. If we multiply problem (34) by a function $\phi \in C_{0}^{\infty}\left(Q_{T}\right)$, we obtain

$$
-\int_{Q_{T}} u_{n} \phi_{t}+\int_{Q_{T}} \mathbf{a}_{n}\left(x, t, u_{n}\right) \nabla u_{n} \nabla \phi=\int_{Q_{T}} T_{n}\left(b\left(u_{n}, \nabla u_{n}\right)\right) \phi+\int_{Q_{T}} T_{n}(f) \phi .
$$

We can easily pass to the limit using (73), (74) and (76). Therefore, we conclude that $u \in C\left([0, T] ; L^{1}(\Omega)\right)$ is a distributional solution of problem (6). The estimates (17) and (18) follow from Proposition 4.1. In the case where the stronger assumption (19) is satisfied for some $\delta>0$, using the estimates (41)-(43) for the approximate solutions $u_{n}$, one easily concludes that

$$
\begin{aligned}
& |\Psi(u)|^{\delta+1} \in L^{2}\left(0, T ; H_{0}^{1}(\Omega)\right) \cap L^{\infty}\left(0, T ; L^{2}(\Omega)\right), \\
& \Phi^{(\delta)}(u) \in L^{\infty}\left(0, T ; L^{1}(\Omega)\right) .
\end{aligned}
$$

This completes the proof of Theorem 2.1 .

\section{Acknowledgements}

This research was begun while Chiara Leone had a post-doc scholarship at the Dipartimento di Metodi e Modelli Matematici of the Università di Roma "La Sapienza", and Sergio Segura de León was visiting professor at the same institution. He wishes to thank for the very warm hospitality enjoyed there. S. Segura de León has been partially supported by the Spanish PNPGC, proyecto BFM2002-01145. 


\section{References}

[1] H.W. Alt, S. Luckhaus, Quasilinear elliptic-parabolic differential equations, Math. Z. 183 (1983) 311-341.

[2] F. Andreu, J.M. Mazón, S. Segura de León, J. Toledo, Existence and uniqueness for a degenerate parabolic equation with $L^{1}$ data, Trans. Amer. Math. Soc. 351 (1999) 285-306.

[3] D.G. Aronson, J. Serrin, Local behavior of solutions of quasilinear parabolic equations, Arch. Rational Mech. Anal. 25 (1967) 81-122.

[4] J.P. Aubin, Un théorème de compacité, C. R. Acad. Sci. 256 (1963) 5042-5044.

[5] P. Bénilan, L. Boccardo, T. Gallouët, R. Gariepy, M. Pierre, J.L. Vázquez, An $L^{1}$ theory of existence and uniqueness of solutions of nonlinear elliptic equations, Ann. Scuola Norm. Sup. Pisa 22 (2) (1995) 240-273.

[6] D. Blanchard, A. Porretta, Nonlinear parabolic equations with natural growth terms and measure initial data, Ann. Scuola Norm. Sup. Pisa Cl. Sci. 30 (4) (2001) 583-622.

[7] L. Boccardo, F. Murat, J.P. Puel, Résultats d'existence pour certains problèmes elliptiques quasilinéaires, Ann. Scuola Norm. Sup. Pisa 11 (1984) 213-235.

[8] L. Boccardo, F. Murat, J.P. Puel, Existence results for some quasilinear parabolic equations, Nonlinear Anal. 13 (1989) 373-392.

[9] L. Boccardo, M.M. Porzio, Bounded solutions for a class of quasi-linear parabolic problems with a quadratic gradient term, Progr. Nonlinear Differential Equations 50 (2002) 39-48.

[10] L. Boccardo, S. Segura de León, C. Trombetti, Bounded and unbounded solutions for a class of quasi-linear elliptic problems with a quadratic grandient term, J. Math. Pures Appl. 80 (9) (2001) 919-940.

[11] H. Brezis, Analyse fonctionnelle. Théorie et applications, Collection Mathématiques Appliquées pour la Maîtrise, Masson, Paris, 1983.

[12] A. Dall'Aglio, D. Giachetti, J.P. Puel, Nonlinear elliptic equations with natural growth in general domains, Ann. Mat. Pura Appl. 181 (2002) 407-426.

[13] A. Dall' Aglio, D. Giachetti, J.P. Puel, Nonlinear parabolic equations with natural growth in general domains, Bull. Un. Mat. Ital., in press.

[14] A. Dall'Aglio, L. Orsina, Nonlinear parabolic equations with natural growth conditions and $L^{1}$ data, Nonlinear Anal. 27 (1996) $59-73$.

[15] E. DiBenedetto, Degenerate Parabolic Equations, Springer-Verlag, New York, 1993.

[16] L.C. Evans, Partial Differential Equations, Graduate Stud. in Math., vol. 19, American Mathematical Society, Providence, RI, 1998.

[17] V. Ferone, F. Murat, Nonlinear problems having natural growth in the gradient: an existence result when the source terms are small, Nonlinear Anal. 42 (2000) 1309-1326.

[18] V. Ferone, M.R. Posteraro, J.M. Rakotoson, $L^{1}$-estimates for nonlinear elliptic problems with $p$-growth in the gradient, J. Ineq. Appl. 3 (1999) 109-125.

[19] V. Ferone, M.R. Posteraro, J.M. Rakotoson, Nonlinear parabolic equations with p-growth and unbounded data, C. R. Acad. Sci. Paris Sèr. I Math. 328 (1999) 291-296.

[20] V. Ferone, M.R. Posteraro, J.M. Rakotoson, Nonlinear parabolic problems with critical growth and unbounded data, Indiana Univ. Math. J. 50 (3) (2001) 1201-1215.

[21] O.A. Ladyzenskaja, V.A. Solonnikov, N.N. Ural'ceva, Linear and Quasi-Linear Equations of Parabolic Type, Transl. Math. Monographs, vol. 23, Amer. Math. Soc., Providence, RI, 1968.

[22] R. Landes, On the existence of weak solutions for quasilinear parabolic boundary value problems, Proc. Roy. Soc. Edinburgh Sect. A 89 (1981) 217-237.

[23] R. Landes, V. Mustonen, On parabolic initial-boundary value problems with critical growth for the gradient, Ann. Inst. H. Poincaré Anal. Non Linéaire 11 (1994) 135-158.

[24] A. Mokrane, Existence of bounded solutions of some nonlinear parabolic equations, Proc. Roy. Soc. Edinburgh Sect. A 107 (3-4) (1987) $313-326$

[25] L. Orsina, M.M. Porzio, $L^{\infty}(Q)$-estimate and existence of solutions for some nonlinear parabolic equations, Boll. Un. Math. Ital. B 6 (1992) 631-647.

[26] A. Porretta, Regularity for entropy solutions of a class of parabolic equations with non regular initial datum, Dynamic Systems Appl. 7 (1998) 53-71.

[27] A. Porretta, S. Segura de León, Nonlinear elliptic equations having a gradient term with natural growth, Preprint.

[28] A. Prignet, Existence and uniqueness of entropy solution of parabolic problems with $L^{1}$ data, Nonlinear Anal. 28 (12) (1997) $1943-1954$.

[29] J. Simon, Compact sets in the space $L^{p}(0, T ; B)$, Ann. Mat. Pura Appl. 146 (1987) 65-96. 